arXiv: arXiv: 1806.09369

\title{
Distance covariance for discretized stochastic processes
}

\author{
Herold Dehling ${ }^{1}$, Muneya Matsui ${ }^{2}$, Thomas Mikosch ${ }^{3}$, Gennady \\ Samorodnitsky $^{4}$ and Laleh Tafakori ${ }^{5}$ \\ ${ }^{1}$ Department of Mathematics, Ruhr-Universität Bochum, 44780 Bochum, Germany e-mail: \\ herold.dehling@rub.de \\ ${ }^{2}$ Department of Business Administration, Nanzan University, 18 Yamazato-cho, Showa-ku, \\ Nagoya 466-8673, Japan e-mail: mmuneya@gmail.com \\ ${ }^{3}$ Department of Mathematics, University of Copenhagen, Universitetsparken 5, DK-2100 \\ Copenhagen, Denmark e-mail: mikosch@math.ku.dk \\ ${ }^{4}$ School of Operations Research and Information \\ Engineering, Cornell University, 220 Rhodes Hall, Ithaca, NY 14853, U.S.A. e-mail: \\ gs18@cornell.edu \\ 5 School of Mathematics and Statistics, University of Melbourne, Richard Berry Building, \\ Parkville, 3010, Melbourne, Australia e-mail: laleh.tafakori@unimelb.edu.au
}

\begin{abstract}
Given an iid sequence of pairs of stochastic processes on the unit interval we construct a measure of independence for the components of the pairs. We define distance covariance and distance correlation based on approximations of the component processes at finitely many discretization points. Assuming that the mesh of the discretization converges to zero as a suitable function of the sample size, we show that the sample distance covariance and correlation converge to limits which are zero if and only if the component processes are independent. To construct a test for independence of the discretized component processes we show consistency of the bootstrap for the corresponding sample distance covariance/correlation.
\end{abstract}

Keywords and phrases: Empirical characteristic function, distance covariance, stochastic process, test of independence.

\section{Introduction}

\subsection{Distance covariance and distance correlation for vectors}

In a series of papers, Székely et al. (2007); Székely and Rizzo (2009, 2013, 2014) introduced distance covariance and distance correlation. They are measures of the dependence between two vectors $\mathbf{X}$ and $\mathbf{Y}$, possibly with different dimensions. These measures have the desirable property that they are zero if and only if $\mathbf{X}$ and $\mathbf{Y}$ are independent. This is in contrast to many other dependence measures where one can only make statements about certain aspects of the dependence between $\mathbf{X}$ and $\mathbf{Y}$. For example, the correlation and covariance between two real-valued random variables $X$ and $Y$ allow one to make statements about their linear dependence. 
The distance covariance between a $p$-dimensional vector $\mathbf{X}$ and a $q$-dimensional vector $\mathbf{Y}$ is a weighted version of the squared distance between the joint characteristic function $\varphi_{\mathbf{X}, \mathbf{Y}}$ of $\mathbf{X}, \mathbf{Y}$ and the product of the marginal characteristic functions $\varphi_{\mathbf{X}}, \varphi_{\mathbf{Y}}$ of these vectors. We know that $\mathbf{X}$ and $\mathbf{Y}$ are independent if and only if

$$
\varphi_{\mathbf{X}, \mathbf{Y}}(\mathbf{s}, \mathbf{t})=\varphi_{\mathbf{X}}(\mathbf{s}) \varphi_{\mathbf{Y}}(\mathbf{t}), \quad \mathbf{s} \in \mathbb{R}^{p}, \mathbf{t} \in \mathbb{R}^{q} .
$$

However, this identity is difficult to check if one has data at the disposal; a replacement of the corresponding characteristic functions by empirical versions does not lead to powerful statistical tools for detecting independence between $\mathbf{X}$ and Y. First, Feuerverger (1993) in the univariate case and, later, Székely et al. (2007); Székely and Rizzo $(2009,2013,2014)$ in the general multivariate case recommended to use a weighted $L^{2}$-distance between $\varphi_{\mathbf{X}, \mathbf{Y}}$ and $\varphi_{\mathbf{X}} \varphi_{\mathbf{Y}}$ : for $\beta \in(0,2)$, the distance covariance between $\mathbf{X}$ and $\mathbf{Y}$ is given by

$$
T_{\beta}(\mathbf{X}, \mathbf{Y})=c_{p} c_{q} \int_{\mathbb{R}^{p+q}}\left|\varphi_{\mathbf{X}, \mathbf{Y}}(\mathbf{s}, \mathbf{t})-\varphi_{\mathbf{X}}(\mathbf{s}) \varphi_{\mathbf{Y}}(\mathbf{t})\right|^{2}|\mathbf{s}|^{-(p+\beta)}|\mathbf{t}|^{-(q+\beta)} d \mathbf{s} d \mathbf{t}
$$

where the constants $c_{d}$ for $d \geq 1$ are chosen such that

$$
c_{d} \int_{\mathbb{R}^{d}}\left(1-\cos \left(\mathbf{s}^{\prime} \mathbf{x}\right)\right)|\mathbf{x}|^{-(d+\beta)} d \mathbf{x}=|\mathbf{s}|^{\beta} .
$$

Here and in what follows we suppress the dependence of the Euclidean norm $|\cdot|$ on the dimension; it will always be clear from the context what the dimension is. The quantity $T_{\beta}(\mathbf{X}, \mathbf{Y})$ is finite under suitable moment conditions on $\mathbf{X}, \mathbf{Y}$. The corresponding distance correlation is given by

$$
R_{\beta}(\mathbf{X}, \mathbf{Y})=\frac{T_{\beta}(\mathbf{X}, \mathbf{Y})}{\sqrt{T_{\beta}(\mathbf{X}, \mathbf{X})} \sqrt{T_{\beta}(\mathbf{Y}, \mathbf{Y})}}
$$

An advantage of choosing the particular weight function $|\mathbf{s}|^{-(p+\beta)}|\mathbf{t}|^{-(q+\beta)}$ is that the distance covariance has an explicit form: for iid copies $\left(\mathbf{X}_{i}, \mathbf{Y}_{i}\right)$, $i=1,2, \ldots$, of $(\mathbf{X}, \mathbf{Y})$ we have

$$
\begin{aligned}
T_{\beta}(\mathbf{X}, \mathbf{Y})= & \mathbb{E}\left[\left|\mathbf{X}_{1}-\mathbf{X}_{2}\right|^{\beta}\left|\mathbf{Y}_{1}-\mathbf{Y}_{2}\right|^{\beta}\right]+\mathbb{E}\left[\left|\mathbf{X}_{1}-\mathbf{X}_{2}\right|^{\beta}\right] \mathbb{E}\left[\left|\mathbf{Y}_{1}-\mathbf{Y}_{2}\right|^{\beta}\right] \\
& -2 \mathbb{E}\left[\left|\mathbf{X}_{1}-\mathbf{X}_{2}\right|^{\beta}\left|\mathbf{Y}_{1}-\mathbf{Y}_{3}\right|^{\beta}\right] .
\end{aligned}
$$

The weight function ensures that $T_{\beta}(c \mathbf{X}, c \mathbf{Y})=c^{2 \beta} T_{\beta}(\mathbf{X}, \mathbf{Y})$ for any constant $c$, hence $R_{\beta}(c \mathbf{X}, c \mathbf{Y})$ does not depend on $c$, i.e., the distance correlation is scale invariant. A corresponding theory can be built on non-homogeneous kernels as well; see the discussion and references in Davis et al. (2018) who consider autoand cross-distance correlation functions for time series.

It is clear from the construction that $T_{\beta}(\mathbf{X}, \mathbf{Y})=R_{\beta}(\mathbf{X}, \mathbf{Y})=0$ if and only if (1.1) holds. This observation motivates the construction of sample versions of $T_{\beta}(\mathbf{X}, \mathbf{Y})$ and $R_{\beta}(\mathbf{X}, \mathbf{Y})$ and one hopes that these have properties similar to 
their deterministic counterparts. In particular, one would like to test independence between $\mathbf{X}$ and $\mathbf{Y}$.

Replacing the characteristic functions in $T_{\beta}(\mathbf{X}, \mathbf{Y})$ and $R_{\beta}(\mathbf{X}, \mathbf{Y})$ by their sample analogs and taking into account (1.2), we obtain the sample versions of $T_{\beta}(\mathbf{X}, \mathbf{Y})$ and $R_{\beta}(\mathbf{X}, \mathbf{Y})$ :

$$
\begin{aligned}
T_{n, \beta}(\mathbf{X}, \mathbf{Y})= & \frac{1}{n^{2}} \sum_{k, l=1}^{n}\left|\mathbf{X}_{k}-\mathbf{X}_{l}\right|^{\beta}\left|\mathbf{Y}_{k}-\mathbf{Y}_{l}\right|^{\beta} \\
& +\frac{1}{n^{2}} \sum_{k, l=1}^{n}\left|\mathbf{X}_{k}-\mathbf{X}_{l}\right|^{\beta} \frac{1}{n^{2}} \sum_{k, l=1}^{n}\left|\mathbf{Y}_{k}-\mathbf{Y}_{l}\right|^{\beta} \\
& -2 \frac{1}{n^{3}} \sum_{k, l, m=1}^{n}\left|\mathbf{X}_{k}-\mathbf{X}_{l}\right|^{\beta}\left|\mathbf{Y}_{k}-\mathbf{Y}_{m}\right|^{\beta} \\
R_{n, \beta}(\mathbf{X}, \mathbf{Y})= & \frac{T_{n, \beta}(\mathbf{X}, \mathbf{Y})}{\sqrt{T_{n, \beta}(\mathbf{X}, \mathbf{X})} \sqrt{T_{n, \beta}(\mathbf{Y}, \mathbf{Y})}}
\end{aligned}
$$

The quantity $T_{n, \beta}(\mathbf{X}, \mathbf{Y})$ is a $V$-statistic; cf. Székely et al. (2007), Lyons (2013). Therefore standard theory yields a.s. consistency,

$$
T_{n, \beta}(\mathbf{X}, \mathbf{Y}) \stackrel{\text { a.s. }}{\rightarrow} T_{\beta}(\mathbf{X}, \mathbf{Y}), \quad n \rightarrow \infty
$$

under suitable moment conditions; see Hoffmann-Jørgensen (1994), Serfling (1980). If $\mathbf{X}$ and $\mathbf{Y}$ are independent the $V$-statistic $T_{n, \beta}(\mathbf{X}, \mathbf{Y})$ is degenerate of order 1. Under suitable moment conditions, one also has the weak convergence of $n T_{n, \beta}(\mathbf{X}, \mathbf{Y})$ to a weighted sum of iid $\chi^{2}$-variables; see Serfling (1980), Lyons (2013), Arcones and Giné (1992). Moreover, $V$-statistics theory also ensures that $T_{n, \beta}(\mathbf{X}, \mathbf{X}) \stackrel{\text { a.s. }}{\rightarrow} T_{\beta}(\mathbf{X}, \mathbf{X})$ and $T_{n, \beta}(\mathbf{Y}, \mathbf{Y}) \stackrel{\text { a.s. }}{\rightarrow} T_{\beta}(\mathbf{Y}, \mathbf{Y})$. Hence $R_{n, \beta}(\mathbf{X}, \mathbf{Y})$ is an a.s. consistent estimator of $R_{\beta}(\mathbf{X}, \mathbf{Y})$ and, modulo a change of scale, $n R_{n, \beta}(\mathbf{X}, \mathbf{Y})$ has the same weak limit as $T_{n, \beta}(\mathbf{X}, \mathbf{Y})$.

\subsection{Distance covariance and distance correlation for stochastic processes}

Székely and Rizzo (2013) considered the situation when $\mathbf{X}$ and $\mathbf{Y}$ are independent and have iid components, $n$ is fixed, $p=q \rightarrow \infty$. Under these conditions, $R_{n, \beta}(\mathbf{X}, \mathbf{Y})$ converges to 1 . In this way, they justified the empirical observation that $R_{n, \beta}(\mathbf{X}, \mathbf{Y})$ is close to 1 if $p, q$ are large relative to $n$.

Matsui et al. (2017) considered a version of the distance covariance for stochastic processes $X, Y$ on $[0,1]$, where it was assumed that the two processes are observed at a Poisson number of points in $[0,1]$. Via simulations the resulting estimator was compared with the distance correlation $R_{n, \beta}(\mathbf{X}, \mathbf{Y})$ where the components of the iid vectors $\left(\mathbf{X}_{i}, \mathbf{Y}_{i}\right)$ consist of a Poisson number of the discretizations of $\left(X_{i}, Y_{i}\right)$, respectively. Both types of estimators exhibited a similar behavior for independent $X$ and $Y$, approaching zero for moderate sizes $n, p, q$.

imsart-generic ver. 2014/10/16 file: dmmsl2018main_2ndsubmissionArxiv.tex date: November 30, 2018 
A possible explanation for this phenomenon is that Matsui et al. (2017) and Székely and Rizzo (2013) worked under quite distinct conditions. Székely and Rizzo (2013) considered vectors $\mathbf{X}$ and $\mathbf{Y}$ with iid components whose dimensions increase to infinity for a fixed sample size $n$. In Matsui et al. (2017), X and $\mathbf{Y}$ can be understood as vectors of discretizations of genuine stochastic processes $X, Y$ on $[0,1]$, such as Brownian motion, fractional Brownian motion, Lévy processes, etc. In these cases, the components of $\mathbf{X}_{i}$ and $\mathbf{Y}_{i}$ are dependent.

In this paper, we again take up the theme of Székely and Rizzo (2013) and Matsui et al. (2017). We consider two processes $X$ and $Y$ on $[0,1]$, which we assume to be stochastically continuous, measurable and bounded. In contrast to Matsui et al. (2017),

- we consider discretizations of these processes at a partition $0=t_{0}<t_{1}<$ $\cdots<t_{p}=1$ of $[0,1]$, assuming that $p=p_{n} \rightarrow \infty$ as $n \rightarrow \infty$ and the mesh satisfies

$$
\delta_{n}=\max _{i=1, \ldots, p}\left(t_{i}-t_{i-1}\right) \rightarrow 0, \quad n \rightarrow \infty,
$$

- we normalize the points $X\left(t_{i}\right)$ and $Y\left(t_{i}\right)$ by $\sqrt{t_{i}-t_{i-1}}$.

In the sequel, we suppress the dependence of $p$ on $n$. It will be convenient to write for any partition $\left(t_{i}\right)$ and a process $Z$ on $[0,1]$,

$\Delta_{i}=\left(t_{i-1}, t_{i}\right],\left|\Delta_{i}\right|=t_{i}-t_{i-1}, i=1, \ldots, p, \Delta Z(s, t]=Z(t)-Z(s), s<t$.

We consider a vector of weighted discretizations

$$
\mathbf{Z}_{p}=\left(\left|\Delta_{1}\right|^{1 / 2} Z\left(t_{1}\right), \ldots,\left|\Delta_{p}\right|^{1 / 2} Z\left(t_{p}\right)\right),
$$

and define

$$
Z^{(p)}(t)=\sum_{i=1}^{p} Z\left(t_{i}\right) \mathbf{1}\left(t \in \Delta_{i}\right), \quad t \in[0,1] .
$$

For stochastically continuous, measurable and bounded processes $Z$ and $Z^{\prime}$ we have

$$
\begin{aligned}
\left|\mathbf{Z}_{p}-\mathbf{Z}_{p}^{\prime}\right|^{2} & =\sum_{i=1}^{p}\left(Z\left(t_{i}\right)-Z^{\prime}\left(t_{i}\right)\right)^{2}\left|\Delta_{i}\right|=\left\|Z^{(p)}-\left(Z^{\prime}\right)^{(p)}\right\|_{2}^{2} \\
& \rightarrow \int_{0}^{1}\left(Z(t)-Z^{\prime}(t)\right)^{2} d t=\left\|Z-Z^{\prime}\right\|_{2}^{2}, \quad p \rightarrow \infty,
\end{aligned}
$$

in probability, where $\|\xi\|_{2}$ denotes the $L^{2}$-norm of a process $\xi$ on $[0,1]$.

For $\beta \in(0,2]$, we introduce a stochastic process analog $T_{\beta}(X, Y)$ of $T_{\beta}(\mathbf{X}, \mathbf{Y})$ from (1.2). Consider an iid sequence $\left(X_{i}, Y_{i}\right), i=1,2, \ldots$, of processes $X_{i}, Y_{i}$ on $[0,1]$ with generic element $(X, Y)$ which is also stochastically continuous, measurable and bounded. Define

$$
T_{\beta}(X, Y)=\mathbb{E}\left[\left\|X_{1}-X_{2}\right\|_{2}^{\beta}\left\|Y_{1}-Y_{2}\right\|_{2}^{\beta}\right]+\mathbb{E}\left[\left\|X_{1}-X_{2}\right\|_{2}^{\beta}\right] \mathbb{E}\left[\left\|Y_{1}-Y_{2}\right\|_{2}^{\beta}\right]
$$




$$
-2 \mathbb{E}\left[\left\|X_{1}-X_{2}\right\|_{2}^{\beta}\left\|Y_{1}-Y_{3}\right\|_{2}^{\beta}\right],
$$

where we assume that all moments involved are finite. Of course, $T_{\beta}(X, Y)=0$ for independent $X, Y$. The converse is not obvious; we prove it in Section 4.

The sample analog of $T_{\beta}(X, Y)$ is given by

$$
\begin{aligned}
T_{n, \beta}(X, Y)= & \frac{1}{n^{2}} \sum_{k, l=1}^{n}\left\|X_{k}-X_{l}\right\|_{2}^{\beta}\left\|Y_{k}-Y_{l}\right\|_{2}^{\beta} \\
& +\frac{1}{n^{2}} \sum_{k, l=1}^{n}\left\|X_{k}-X_{l}\right\|_{2}^{\beta} \frac{1}{n^{2}} \sum_{k, l=1}^{n}\left\|Y_{k}-Y_{l}\right\|_{2}^{\beta} \\
& -2 \frac{1}{n^{3}} \sum_{k, l, m=1}^{n}\left\|X_{k}-X_{l}\right\|_{2}^{\beta}\left\|Y_{k}-Y_{m}\right\|_{2}^{\beta} \\
=: & \mathrm{I}_{1}+\mathrm{I}_{3}-2 \mathrm{I}_{2} .
\end{aligned}
$$

Assuming that the moments in $T_{\beta}(X, Y)$ are finite, the strong law of large numbers for $V$-statistics yields

$$
T_{n, \beta}(X, Y) \stackrel{\text { a.s. }}{\rightarrow} T_{\beta}(X, Y), \quad n \rightarrow \infty .
$$

This fact and the observation that $T_{\beta}(X, Y)$ vanishes for independent $X, Y$ encourage one to call $T_{\beta}(X, Y)$ the distance covariance between $X, Y$, and $T_{n, \beta}(X, Y)$ its sample version. The corresponding distance and sample distance correlations $R_{\beta}(X, Y)$ and $R_{n, \beta}(X, Y)$ are defined in the natural way.

\subsection{Objectives}

We imagine that the coastline of a country (like the Netherlands) can be mapped to the interval $[0,1]$ and, at each location $s \in[0,1]$ and on each day $i$, we have an observation of the height of sea waves, $X_{i}$, and the corresponding wind-speed, $Y_{i}$. An interesting question is whether the processes $X_{i}$ and $Y_{i}$ are independent. Similarly, we can think of two price processes $X_{i}$ and $Y_{i}$ on day $i$ given on the interval of the working hours of the stock exchange. Natural questions are as to whether the two price processes are independent on the same day and how much serial dependence there is in each of the series $\left(X_{i}\right)$ and $\left(Y_{i}\right)$ and between them. In the first case, one is interested in testing the independence of the processes $X_{i}$ and $Y_{i}$. In the second case, one is interested in testing the independence of $X_{i}$ and $X_{i+h}, Y_{i+h}$ for positive lags.

Typically, we will not have complete sample paths of $\left(X_{i}, Y_{i}\right)$ at our disposal. In this paper, we assume that we observe a sample $\left(\left(X_{i}^{(p)}, Y_{i}^{(p)}\right)\right)_{i=1, \ldots, n}$ consisting of discretizations taken from an iid sequence $\left(\left(X_{i}, Y_{i}\right)\right)_{i=1,2, \ldots}$ on the same partition $\left(t_{i}\right)_{i=0, \ldots, p}$ of $[0,1]$. We can define the corresponding sample distance covariance $T_{n, \beta}\left(X^{(p)}, Y^{(p)}\right)$ and sample distance correlation $R_{n, \beta}\left(X^{(p)}, Y^{(p)}\right)$. In view of the discussion above we see that the latter quantities coincide with the 
corresponding quantities $T_{n, \beta}\left(\mathbf{X}_{p}, \mathbf{Y}_{p}\right)$ and $R_{n, \beta}\left(\mathbf{X}_{p}, \mathbf{Y}_{p}\right)$ where $\mathbf{X}_{p}$ and $\mathbf{Y}_{p}$ are defined through (1.3). In the case of an equidistant partition with mesh $\delta_{n}=1 / p$ we also observe that $R_{n, \beta}\left(\mathbf{X}_{p}, \mathbf{Y}_{p}\right)$ is exactly the classical sample distance correlation $R_{n, \beta}(\mathbf{X}, \mathbf{Y})$ of the vectors $\mathbf{X}=(X(j / p))_{j=1, \ldots, p}$ and $\mathbf{Y}=(Y(j / p))_{j=1, \ldots, p}$.

The main goal of this paper is to show that for independent $X, Y$,

$$
n\left(T_{n, \beta}\left(X^{(p)}, Y^{(p)}\right)-T_{n, \beta}(X, Y)\right) \stackrel{\mathbb{P}}{\rightarrow} 0, \quad n \rightarrow \infty,
$$

provided $\delta_{n} \rightarrow 0$ and $p=p_{n} \rightarrow \infty$ sufficiently fast. In turn, we will be able to exploit the existing limit theory for the normalized degenerate $V$-statistic $n T_{n, \beta}(X, Y)$ to derive the distributional limit of $n T_{n, \beta}\left(X^{(p)}, Y^{(p)}\right)$. This limit has a weighted $\chi^{2}$-distribution which is not easily evaluated. We will show that bootstrap versions of the degenerate $V$-statistics $n T_{n, \beta}(X, Y)$ and $n T_{n, \beta}\left(X^{(p)}, Y^{(p)}\right)$ are close in the sense of Mallows metrics and have the same distributional limit as $n T_{n, \beta}(X, Y)$.

The paper is organized as follows. In Section 2 we introduce various technical conditions and discuss their applicability to some classes of stochastic processes. The main results of Theorem 3.1 yield sufficient conditions for (1.6) and the corresponding versions for the distance correlations, assuming independence between $X, Y$. The proof is given in Section 7 . The bootstrap for $T_{n, \beta}\left(X^{(p)}, Y^{(p)}\right)$ is discussed in Section 5. There we show that a suitable bootstrap version of $T_{n, \beta}\left(X^{(p)}, Y^{(p)}\right)$ is consistent. The results of Section 4 may be of independent interest. There we show that $T_{\beta}(X, Y)=0$ implies independence of the integrals $\int X d B_{1}$ and $\int Y d B_{2}$ conditional on $B=\left(B_{1}, B_{2}\right)$ which has independent Brownian motion components on $[0,1]$ and is independent of $(X, Y)$. In turn, the conditional independence of these integrals implies independence of $X, Y$. We give a small simulation study in Section 6 which shows that the theoretical results work for small and moderate values of $n$ and $p$.

\section{Technical conditions}

To derive the results in Section 3 we assume various conditions on the smoothness and moments of the processes $X, Y$ and their relation with the parameters of the partition, in particular $p$ and $\delta_{n}$. Throughout $\beta \in(0,2)$ is fixed. If any of the processes $X, Y$ have finite expectation we assume that they are centered.

We will work under two distinct settings: (1) finite variance of $X, Y$ and (2) $X, Y$ have finite $\beta$ th moment.

\subsection{The finite variance case}

If $X, Y$ have finite second moments we will work under the set of conditions (A):

(A1) Smoothness of increments. There exist $\gamma_{X}, \gamma_{Y}>0$ and $c>0$ such that $\operatorname{var}(\Delta X(s, t]) \leq c|t-s|^{\gamma_{X}} \quad$ and $\quad \operatorname{var}(\Delta Y(s, t]) \leq c|t-s|^{\gamma_{Y}}, \quad s<t$. 
(A2) Growth condition on $p=p_{n} \rightarrow \infty$. We have

$$
\delta_{n}=o\left(n^{-2 /\left(\left(\gamma_{X} \wedge \gamma_{Y}\right)(\beta \wedge 1)\right)}\right), \quad n \rightarrow \infty .
$$

(A3) Additional moment conditions. If $\beta \in(1,2)$ we have

$$
\max _{0 \leq t \leq 1} \mathbb{E}\left[|X(t)|^{2(2 \beta-1)}\right]+\max _{0 \leq t \leq 1} \mathbb{E}\left[|Y(t)|^{2(2 \beta-1)}\right]<\infty .
$$

\subsection{The finite $\beta$ th moment case}

If $X, Y$ possibly have infinite second moments we will work under the set of conditions (B):

(B1) Finite $\beta$ th moment.

$$
\mathbb{E}\left[\max _{t \in(0,1]}|X(t)|^{\beta}\right]<\infty \text { and } \mathbb{E}\left[\max _{t \in(0,1]}|Y(t)|^{\beta}\right]<\infty,
$$

(B2) Smoothness of increments. There exist $\gamma_{X}, \gamma_{Y}>0$ and $c>0$ such that

$$
\max _{i=1, \ldots, p} \mathbb{E}\left[\max _{t \in \Delta_{i}}\left|\Delta X\left(t, t_{i}\right]\right|^{\beta}\right] \leq c \delta_{n}^{\gamma_{X}} \text { and } \max _{i=1, \ldots, p} \mathbb{E}\left[\max _{t \in \Delta_{i}}\left|\Delta Y\left(t, t_{i}\right]\right|^{\beta}\right] \leq c \delta_{n}^{\gamma_{Y}} .
$$

(B3) Additional moment and smoothness conditions. If $\beta \in(0,1)$ we also have

$$
\mathbb{E}\left[\max _{0 \leq t \leq 1}|X(t)|^{2 \beta}\right]<\infty \text { and } \mathbb{E}\left[\max _{0 \leq t \leq 1}|Y(t)|^{2 \beta}\right]<\infty,
$$

and there exist $\gamma_{X}^{\prime}, \gamma_{Y}^{\prime}>0$ and $c>0$ such that

$$
\max _{i=1, \ldots, p} \mathbb{E}\left[\max _{t \in \Delta_{i}}\left|\Delta X\left(t, t_{i}\right]\right|^{2 \beta}\right] \leq c \delta_{n}^{\gamma_{X}^{\prime}} \text { and } \max _{i=1, \ldots, p} \mathbb{E}\left[\max _{t \in \Delta_{i}}\left|\Delta Y\left(t, t_{i}\right]\right|^{2 \beta}\right] \leq c \delta_{n}^{\gamma_{Y}^{\prime}} .
$$

(B4) Growth condition on $p=p_{n} \rightarrow \infty$. We have

$$
\delta_{n}=o\left(\left(p n^{\beta /(\beta \wedge 1)}\right)^{-\frac{1}{\beta / 2+\gamma^{\wedge} \gamma_{Y}}}\right) .
$$

\subsection{Discussion of the conditions and examples}

Remark 2.1. In the proofs we will need the conditions

$$
\mathbb{E}\left[\|X\|_{2}^{\beta}\right]<\infty \text { and } \mathbb{E}\left[\|Y\|_{2}^{\beta}\right]<\infty \text { for some } \beta \in(0,2) .
$$

If (A1) holds (in particular, $\left.\sup _{t \in[0,1]}[\operatorname{var}(X(t))+\operatorname{var}(Y(t))]<\infty\right)(2.1)$ is automatic because by Jensen's inequality

$$
\mathbb{E}\left[\|X\|_{2}^{\beta}\right]=\mathbb{E}\left[\left(\int_{0}^{1}(X(t))^{2} d t\right)^{\beta / 2}\right] \leq\left(\int_{0}^{1} \operatorname{var}(X(t)) d t\right)^{\beta / 2}<\infty .
$$

The same argument also shows that $\mathbb{E}\left[\|X\|_{2}^{2}\right]<\infty$ under (A1). If (B1) holds then (2.1) follows. 
Remark 2.2. In the case of an equidistant partition we have $\delta_{n}=1 / p$. Then the growth condition (A2) reads as

$$
\frac{p}{n^{\frac{2}{\left(\gamma^{\wedge} \gamma_{Y}\right)(\beta \wedge 1)}}} \rightarrow \infty, \quad n \rightarrow \infty,
$$

while (B4) takes on the form

$$
\frac{p}{n^{\frac{\beta}{\left(\beta / 2+\gamma_{X} \wedge \gamma_{Y}-1\right)(\beta \wedge 1)}}} \rightarrow \infty, \quad n \rightarrow \infty,
$$

provided one can ensure that $\beta / 2+\gamma_{X} \wedge \gamma_{Y}>1$. The message from (2.2) is that we need to choose $p$ the larger the smaller $\gamma_{X} \wedge \gamma_{Y}$ is, i.e., the rougher the sample paths. Similarly, for $\beta<1, p$ needs to be chosen the larger the smaller $\beta$ is. Similar comments apply to $(2.3)$.

Example 2.3. Assume that $X, Y$ are sample continuous self-similar processes with stationary increments and a finite variance. If the corresponding Hurst exponents are $H_{X}, H_{Y} \in(0,1)$ then for some $c_{X}>0$,

$$
\operatorname{var}(\Delta X(s, t])=\operatorname{var}(X(0, t-s])=c_{X}(t-s)^{2 H_{X}}, \quad s<t,
$$

and similarly for $Y$. That is, we can choose $\gamma_{X}=2 H_{X}$ and $\gamma_{Y}=2 H_{Y}$ in (A1). Furthermore, (A3) holds for $X$ if $\beta \in(1,2)$ and $\mathbb{E}\left[|X(1)|^{2(2 \beta-1)}\right]<\infty$, and similarly for $Y$. A special case is that of Gaussian $X$ and $Y$ which then are fractional Brownian motions, and (A3) trivially holds. A process with the same covariance structure is the fractional Lévy process

$$
X(t)=\int_{\mathbb{R}}\left((t-s)_{+}^{H_{X}-0.5}-(-s)_{+}^{H_{X}-0.5}\right) d L(s), \quad t \in \mathbb{R}, H_{X} \in(0.5,1),
$$

where $L$ is a two-sided Lévy process on $\mathbb{R}$ with mean zero and finite variance, introduced in Marquardt (2006). This process is not self-similar (unless $L$ is a Brownian motion) but has stationary increments. Here (A1) holds with $\gamma_{X}=$ $2 H_{X}$ and $\gamma_{Y}=2 H_{Y}$. Furthermore, (A3) holds if $\mathbb{E}\left[|L(1)|^{2(2 \beta-1)}\right]<\infty$.

Notice also that any centered Gaussian processes $X$ and $Y$ satisfying (A1) have automatically continuous sample paths and (A3) is satisfied.

Example 2.4. Assume that $X$ and $Y$ are Itô integrals, i.e., there are two Brownian motions $B_{X}, B_{Y}$ and predictable processes $Z_{X}, Z_{Y}$ with respect to the corresponding Brownian filtrations such that

$$
X(t)=\int_{0}^{t} Z_{X}(s) d B_{X}(s), \quad Y(t)=\int_{0}^{t} Z_{Y}(s) d B_{Y}(s), \quad 0 \leq t \leq 1 .
$$

Then we have

$$
\operatorname{var}(\Delta X(s, t])=\int_{s}^{t} \mathbb{E}\left[Z_{X}^{2}(x)\right] d x, \quad s<t
$$


Hence, if $c_{X}=\sup _{x \in[0,1]} \mathbb{E}\left[Z_{X}^{2}(x)\right]<\infty$, then $\operatorname{var}(\Delta X(s, t]) \leq c_{X}(t-s)$, and one can choose $\gamma_{X}=1$ in (A1). Moreover, (A3) holds for $X$ if $\beta \in(1,2)$ and $\mathbb{E}\left[|X(1)|^{2(2 \beta-1)}\right]<\infty$. This follows from an application of Doob's maximal inequality for martingales. Similar arguments apply to the process $Y$. A special case is that of zero drift geometric Brownian motions; a simple computation shows that nothing changes even when the drift is not zero.

In the equidistant case we conclude from (2.2) that (A2) holds if

$$
\frac{p}{n^{\frac{2}{\beta \wedge 1}}} \rightarrow \infty, \quad n \rightarrow \infty .
$$

Example 2.5. For $\alpha \in(0,2)$ sample continuous self-similar $\mathrm{S} \alpha \mathrm{S}$ processes with stationary increments provide a family of examples with an infinite second moment. For such processes (B1) is satisfied for $\beta<\alpha$ and (B2) is satisfied with $\gamma_{X}=\gamma_{Y}=\beta H$, where $H$ is the Hurst exponent. This follows from continuity, self-similarity and stationarity of the increments. Similarly, (B3) holds if $\beta<\alpha / 2$ and $\gamma_{X}^{\prime}=\gamma_{Y}^{\prime}=2 \beta H$. Such processes include the fractional harmonizable $\alpha$-stable motions and, if $1<\alpha<2$ and $1 / \alpha<H<1$, also the linear fractional stable motions; see Chapter 7 in Samorodnitsky and Taqqu (1994). Another example is that of the $\gamma$-Mittag Leffler fractional SaS motion, which is an integral of a $\gamma$-Mittag Leffler process with respect to a suitable $\mathrm{S} \alpha \mathrm{S}$ random measure; see Samorodnitsky (2016), Section 8.4. Here $H=\gamma+(1-\gamma) / \alpha$.

Example 2.6. Lévy processes are stochastically continuous and bounded by definition. If $X$ is a Lévy process with finite second moment (A1) holds because $\operatorname{var}(\Delta X(s, t))=c(t-s)$, for $s<t$ and a constant $c$. Moreover, (A3) holds for

$X$ if $\mathbb{E}\left[|X(1)|^{2(2 \beta-1)}\right]<\infty$. Indeed, an application of Lévy's maximal inequality yields for $t \in[0,1]$,

$$
\mathbb{E}\left[|X(t)|^{2(2 \beta-1)}\right] \leq \mathbb{E}\left[\max _{0 \leq t \leq 1}|X(t)|^{2(2 \beta-1)}\right] \leq c \mathbb{E}\left[|X(1)|^{2(2 \beta-1)}\right] .
$$

Similarly, for $X$, (B1) holds if $\mathbb{E}\left[|X(1)|^{\beta}\right]<\infty,(\mathrm{B} 2)$ is satisfied if $\mathbb{E}\left[|\Delta X(s, t]|^{\beta}\right] \leq$ $c(t-s)^{\gamma_{X}}$, and (B3) holds if $\mathbb{E}\left[|\Delta X(s, t]|^{2 \beta}\right] \leq c(t-s)^{\gamma_{X}^{\prime}}$.

\section{Main results}

We would like to use the distance covariance to test for independence of two stochastically continuous bounded stochastic processes $X, Y$ on $[0,1]$. By the strong law of large numbers for $V$-statistics we have

$$
T_{n, \beta}(X, Y) \stackrel{\text { a.s. }}{\rightarrow} T_{\beta}(X, Y),
$$

where the limit is defined in (1.4). If $X, Y$ are independent then $T_{\beta}(X, Y)=0$, and in Section 4 we prove that, conversely, $T_{\beta}(X, Y)=0$ implies independence of $X, Y$. The following theorem establishes, in particular, that under appropriate conditions, if $X, Y$ are independent, then also

$$
T_{n, \beta}\left(X^{(p)}, Y^{(p)}\right)-T_{n, \beta}(X, Y) \stackrel{\mathbb{P}}{\rightarrow} 0
$$


and, hence,

$$
T_{n, \beta}\left(X^{(p)}, Y^{(p)}\right) \stackrel{\mathbb{P}}{\rightarrow} 0
$$

This relation can be used in testing for independence of $X, Y$. Note that, if $X, Y$ are dependent the results of Section 4 will imply that $T_{\beta}(X, Y)>0$ and so, by (3.1) and (3.2), we see that $n T_{n, \beta}\left(X^{(p)}, Y^{(p)}\right) \stackrel{\mathbb{P}}{\rightarrow} \infty$.

In fact, the limiting equivalence (3.2) holds for dependent $X, Y$ as well, see the proof of Lemma 7.2, as long as one imposes more restrictive moment conditions (due to the use of Hölder-type inequalities for products of dependent random variables).

In the theorem below we assume, without loss of generality, that $\mathbb{E}[X(t)]=$ $\mathbb{E}[Y(t)]=0$ for any $t \in[0,1]$, provided the expectations are finite. Indeed, $T_{n, \beta}$ contains expressions of the type $X_{k}-X_{l}, Y_{k}-Y_{l}$ or their discrete approximations. Therefore we can always mean-correct $X_{k}$ and $Y_{k}$, without changing the value of $T_{n, \beta}$.

Theorem 3.1. Assume the following conditions:

1. $X, Y$ are independent stochastically continuous bounded processes on $[0,1]$ defined on the same probability space.

2. If $X, Y$ have finite expectations, then these are assumed to be equal to 0 .

3. $\delta_{n} \rightarrow 0$ as $n \rightarrow \infty$.

4. $\beta \in(0,2)$.

Then the following statements hold.

(1) If either (A1) or [(B1),(B2) and $\left.p \delta_{n}^{\beta / 2+\gamma_{X} \wedge \gamma_{Y}} \rightarrow 0\right]$ are satisfied then (3.2) (and, hence, (3.3)) hold.

(2) If either (A1),(A2) or (B1),(B2),(B4) hold then

$$
n T_{n, \beta}\left(X^{(p)}, Y^{(p)}\right) \stackrel{d}{\rightarrow} \sum_{i=1}^{\infty} \lambda_{i}\left(N_{i}^{2}-1\right)+c
$$

for an iid sequence of standard normal random variables $\left(N_{i}\right)$, a constant $c$, and a square summable sequence $\left(\lambda_{i}\right)$.

(3) If either (A1),(A3) or $\left[\beta \in(0,1)\right.$ and (B1)-(B3) and $\left.p \delta_{n}^{\beta+\gamma_{X}^{\prime} \wedge \gamma_{Y}^{\prime}} \rightarrow 0\right]$ hold then

$$
R_{n, \beta}\left(X^{(p)}, Y^{(p)}\right) \stackrel{\mathbb{P}}{\rightarrow} 0
$$

(4) If either (A1)-(A3) or $\left[\beta \in(0,1)\right.$ and (B1)-(B4) and $\left.p \delta_{n}^{\beta+\gamma_{X}^{\prime} \wedge \gamma_{Y}^{\prime}} \rightarrow 0\right]$ hold then

$$
n R_{n, \beta}\left(X^{(p)}, Y^{(p)}\right) \stackrel{d}{\rightarrow} \sum_{i=1}^{\infty} \lambda_{i}\left(N_{i}^{2}-1\right)+c
$$

for an iid sequence of standard normal random variables $\left(N_{i}\right)$, a constant $c$, and a square summable sequence $\left(\lambda_{i}\right)$. 
The proof is given in Section 7 .

Remark 3.2. In Appendix $\mathrm{C}$ we discuss the asymptotic behavior of $T_{n, \beta}\left(X^{(p)}, Y^{(p)}\right)$ and $R_{n, \beta}\left(X^{(p)}, Y^{(p)}\right)$ for dependent processes $X, Y$. In this case $T_{\beta}(X, Y)$ is positive. We prove central limit theory with Gaussian limits for

$$
\sqrt{n}\left(T_{n, \beta}\left(X^{(p)}, Y^{(p)}\right)-T_{\beta}(X, Y), R_{n, \beta}\left(X^{(p)}, Y^{(p)}\right)-R_{\beta}(X, Y)\right) .
$$

In particular, if one used the normalization $n$ for the independent case, one would get $n T_{n, \beta}\left(X^{(p)}, Y^{(p)}\right) \stackrel{\mathbb{P}}{\rightarrow} \infty$ and $n R_{n, \beta}\left(X^{(p)}, Y^{(p)}\right) \stackrel{\mathbb{P}}{\rightarrow} \infty$. This observation allows one to clearly distinguish between the independent case and the alternative of dependent $X, Y$.

The distinct asymptotic behavior of $T_{n, \beta}\left(X^{(p)}, Y^{(p)}\right)$ and $R_{n, \beta}\left(X^{(p)}, Y^{(p)}\right)$ in the independent and dependent cases is explained by the $V$-statistic structure underlying the sample distance covariance $T_{n, \beta}\left(X^{(p)}, Y^{(p)}\right)$. Indeed, this quantity is approximated by the non-degenerate $V$-statistic $T_{n, \beta}(X, Y)$. In view of classical limit theory (see Arcones and Giné (1992)) non-degenerate $V$-statistics satisfy the central limit theorem with normalization $\sqrt{n}$.

Remark 3.3. The numbers $\lambda_{i}$ in parts (2) and (4) of the theorem are the eigenvalues of certain integral operators. This follows from limit theory for degenerate $V$-statistics; see Serfling (1980), Lyons (2013), Arcones and Giné (1992). Unfortunately, neither the $\lambda_{i}$ nor the distribution of the limit are available. Arcones and Giné (1992) proved the consistency of a bootstrap version of degenerate $U$ - and $V$-statistics. These latter results apply to $T_{n, \beta}(X, Y)$ but not to $T_{n, \beta}\left(X^{(p)}, Y^{(p)}\right)$. In Section 5 we argue that the bootstrap also works for a modification of the latter quantity.

\section{The condition $T_{\beta}(X, Y)=0$ and independence of $X$ and $Y$}

The results in the previous section tell us that $T_{n, \beta}\left(X^{(p)}, Y^{(p)}\right) \stackrel{\mathbb{P}}{\rightarrow} T_{\beta}(X, Y)=0$ for independent $X, Y$ under various conditions on $X, Y$ and the size of the mesh $\delta_{n}$ of the partition $\left(t_{i}\right)$. An important question is whether, conversely, $T_{\beta}(X, Y)=0$ also implies independence of $X, Y$. In the case $\beta \in(0,1]$ an affirmative answer to this question follows from Lyons (2013), based on the fact that the metric obtained by raising the separable Hilbert space distance to the power $\beta \in(0,1]$ is of the strong negative type. In the sequel we extend the converse statement to all $\beta \in(0,2)$. Our approach is based on studying the conditional independence of certain stochastic integrals.

Let $B_{1}$ and $B_{2}$ be independent Brownian motions on $[0,1]$, independent of a pair $(X, Y)$ of stochastically continuous bounded stochastic processes $[0,1]$. The stochastic integrals

$$
Z_{1}=\int_{0}^{1} X d B_{1} \quad \text { and } \quad Z_{2}=\int_{0}^{1} Y d B_{2}
$$

are well defined (and are, given $(X, Y)$, independent normal random variables). 
The next lemma demonstrates a connection between such stochastic integrals and distance covariances. Let $\mathcal{F}_{B}$ denote the $\sigma$-field generated by $B=\left(B_{1}, B_{2}\right)$.

Lemma 4.1. Let $\beta \in(0,2)$ and assume that $\mathbb{E}\left[\|X\|_{2}^{\beta}\right]+\mathbb{E}\left[\|Y\|_{2}^{\beta}\right]<\infty$. Let $Y^{\prime}$ be a copy of $Y$ independent of everything else. Then

$$
\begin{array}{r}
c_{0}^{2} T_{\beta}(X, Y)=\int_{\mathbb{R}^{2}}|s t|^{-(1+\beta / 2)} \mathbb{E} \mid \mathbb{E}\left[\mathrm{e}^{i s \int X(u) d B_{1}(u)} \mathrm{e}^{i t \int Y(u) d B_{2}(u)}\right. \\
\left.-\mathrm{e}^{i s \int X(u) d B_{1}(u)} \mathrm{e}^{i t \int Y^{\prime}(u) d B_{2}(u)} \mid \mathcal{F}_{B}\right]\left.\right|^{2} d s d t,
\end{array}
$$

where

$$
c_{0}=\int_{\mathbb{R}} \frac{1-\mathrm{e}^{-\frac{s^{2}}{2}}}{|s|^{1+\beta / 2}} d s .
$$

Proof. Consider an independent copy $\left(X^{\prime}, Y^{\prime}\right)$ of $(X, Y)$ and let $Y^{\prime \prime}, Y^{\prime \prime \prime}$ be independent copies of $Y$ which are independent of everything else. The expectation on the right-hand side in (4.1) can be written as

$$
\begin{gathered}
\mathbb{E}\left[\mathrm{e}^{i s \int\left(X-X^{\prime}\right) d B_{1}+i t \int\left(Y-Y^{\prime}\right) d B_{2}}+\mathrm{e}^{i s \int\left(X-X^{\prime}\right) d B_{1}+i t \int\left(Y^{\prime \prime}-Y^{\prime \prime \prime}\right) d B_{2}}\right. \\
\left.-\mathrm{e}^{i s \int\left(X-X^{\prime}\right) d B_{1}-i t \int\left(Y-Y^{\prime \prime}\right) d B_{2}}-\mathrm{e}^{-i s \int\left(X-X^{\prime}\right) d B_{1}+i t \int\left(Y-Y^{\prime \prime}\right) d B_{2}}\right] \\
=\mathbb{E}\left[\mathrm{e}^{-\frac{s^{2}}{2} \int\left(X(u)-X^{\prime}(u)\right)^{2} d u-\frac{t^{2}}{2} \int\left(Y(u)-Y^{\prime}(u)\right)^{2} d u}\right. \\
+\mathrm{e}^{-\frac{s^{2}}{2} \int\left(X(u)-X^{\prime}(u)\right)^{2} d u-\frac{t^{2}}{2} \int\left(Y^{\prime \prime}(u)-Y^{\prime \prime \prime}(u)\right)^{2} d u} \\
\left.-2 \mathrm{e}^{-\frac{s^{2}}{2} \int\left(X(u)-X^{\prime}(u)\right)^{2} d u-\frac{t^{2}}{2} \int\left(Y(u)-Y^{\prime \prime}(u)\right)^{2} d u}\right] \\
\mathbb{E}\left[\left(1-\mathrm{e}^{-\frac{s^{2}}{2} \int\left(X(u)-X^{\prime}(u)\right)^{2} d u}\right)\left(1-\mathrm{e}^{-\frac{t^{2}}{2} \int\left(Y(u)-Y^{\prime}(u)\right)^{2} d u}\right)\right. \\
+\left(1-\mathrm{e}^{-\frac{s^{2}}{2} \int\left(X(u)-X^{\prime}(u)\right)^{2} d u}\right)\left(1-\mathrm{e}^{-\frac{t^{2}}{2} \int\left(Y^{\prime \prime}(u)-Y^{\prime \prime \prime}(u)\right)^{2} d u}\right) \\
\left.-2\left(1-\mathrm{e}^{-\frac{s^{2}}{2} \int\left(X(u)-X^{\prime}(u)\right)^{2} d u}\right)\left(1-\mathrm{e}^{-\frac{t^{2}}{2} \int\left(Y(u)-Y^{\prime \prime}(u)\right)^{2} d u}\right)\right] .
\end{gathered}
$$

By change of variables,

$$
\int_{\mathbb{R}} \frac{1-\mathrm{e}^{-\frac{s^{2}}{2} \int\left(X(u)-X^{\prime}(u)\right)^{2} d u}}{|s|^{1+\beta / 2}} d s=c_{0}\left\|X-X^{\prime}\right\|_{2}^{\beta} .
$$

Thus $T_{\beta}(X, Y)$ coincides with

$\mathbb{E}\left[\left\|X-X^{\prime}\right\|_{2}^{\beta}\left\|Y-Y^{\prime}\right\|_{2}^{\beta}+\left\|X-X^{\prime}\right\|_{2}^{\beta}\left\|Y^{\prime \prime}-Y^{\prime \prime \prime}\right\|_{2}^{\beta}-2\left\|X-X^{\prime}\right\|_{2}^{\beta}\left\|Y-Y^{\prime \prime}\right\|_{2}^{\beta}\right]$.

An immediate corollary of Lemma 4.1 is that $T_{\beta}(X, Y)=0$ implies that, for a.e. $s, t$,

$$
\mathbb{E}\left[\mathrm{e}^{i s \int X(u) d B_{1}(u)} \mathrm{e}^{i t \int Y(u) d B_{2}(u)}-\mathrm{e}^{i s \int X(u) d B_{1}(u)} \mathrm{e}^{i t \int Y^{\prime}(u) d B_{2}(u)} \mid \mathcal{F}_{B}\right]=0
$$

imsart-generic ver. 2014/10/16 file: dmms12018main_2ndsubmissionArxiv.tex date: November 30, 2018 
with probability 1. By Fubini's theorem, on an event of probability 1, this equality holds for all rational $s, t$, hence for all real $s, t$. We conclude that the stochastic integrals $Z_{1}, Z_{2}$ are conditionally independent given $\mathcal{F}_{B}$.

The next theorem, which is the main result of this section, shows that this implies independence of $X$ and $Y$.

Theorem 4.2. If the stochastic integrals $Z_{1}$ and $Z_{2}$ are a.s. conditionally independent given $\mathcal{F}_{B}$ then $X, Y$ are independent. In particular, if $\beta \in(0,2)$ and $\mathbb{E}\left[\|X\|_{2}^{\beta}\right]+\mathbb{E}\left[\|Y\|_{2}^{\beta}\right]<\infty$, then $T_{\beta}(X, Y)=0$ if and only if $X, Y$ are independent.

Proof. Only the fact that the conditional independence of the integrals implies independence of $X$ and $Y$ remains to be proved. Let $(a(t), 0 \leq t \leq 1)$ and $(b(t), 0 \leq t \leq 1)$ be functions in $L^{2}[0,1]$, and

$$
A_{1}(t)=\int_{0}^{t} a(s) d s \quad \text { and } \quad A_{2}(t)=\int_{0}^{t} b(s) d s, \quad 0 \leq t \leq 1 .
$$

Since the law of the bivariate process

$$
\left(\tilde{B}_{1}(t), \tilde{B}_{2}(t), 0 \leq t \leq 1\right)=\left(B_{1}(t)+A_{1}(t), B_{2}(t)+A_{2}(t)\right), \quad 0 \leq t \leq 1,
$$

is equivalent to the law of the standard bivariate Brownian motion, it follows that the integrals

$$
\int_{0}^{1} X(t) d \tilde{B}_{1}(t)=\int_{0}^{1} X(t) d B_{1}(t)+\int_{0}^{1} X(t) a(t) d t
$$

and

$$
\int_{0}^{1} Y(t) d \tilde{B}_{2}(t)=\int_{0}^{1} Y(t) d B_{2}(t)+\int_{0}^{1} Y(t) b(t) d t
$$

are a.s. conditionally independent given $\mathcal{F}_{B}$.

It is not difficult to construct a sequence $\left(C_{n}\right)$ of events in $\mathcal{F}_{B}$, of positive probability, such that the conditional laws of the integrals

$$
\int_{0}^{1} X(t) d B_{1}(t) \text { and } \int_{0}^{1} Y(t) d B_{2}(t)
$$

given $C_{n}$ converge to the degenerate law at zero as $n \rightarrow \infty$. One way for producing such a sequence of events is to let the two independent Brownian motions take values close to zero at the points $i / n, i=0,1, \ldots, n$. Letting $n \rightarrow \infty$ we conclude that the integrals

$$
\int_{0}^{1} X(t) a(t) d t \text { and } \int_{0}^{1} Y(t) b(t) d t
$$

are independent.

For every fixed realization of the processes $X$ and $Y$,

$$
\lim _{\varepsilon \rightarrow 0} \frac{1}{\varepsilon} \int_{t}^{t+\varepsilon} X(s) d s=X(t) \text { and } \lim _{\varepsilon \rightarrow 0} \frac{1}{\varepsilon} \int_{t}^{t+\varepsilon} Y(t) d s=Y(s)
$$


for all $t$ in a set of full Lebesgue measure. By Fubini's theorem there is a set $M$ of full Lebesgue measure such that, for every $t \in M,(4.2)$ holds a.s. By necessity, the set $M$ is dense in $[0,1]$.

To prove our claim it suffices to prove that for any points $0=t_{0}<t_{1}<$ $\cdots<t_{k}<t_{k+1}=1, k \geq 1$, the random vectors $\left(X\left(t_{1}\right), \ldots, X\left(t_{k}\right)\right)$ and $\left(Y\left(t_{1}\right), \ldots, Y\left(t_{k}\right)\right)$ are independent. By stochastic continuity of the processes $X$ and $Y$ it is enough to restrict ourselves to the case when every $t_{i} \in M$. Let $0<\varepsilon<\min _{i=1, \ldots, k}\left(t_{i+1}-t_{i}\right)$. Choosing piece-wise constant functions $(a(t), 0 \leq t \leq 1)$ and $(b(t), 0 \leq t \leq 1)$, we conclude that the sums

$$
\sum_{i=1}^{k} \theta_{i} \int_{t_{i}}^{t_{i}+\varepsilon} X(t) d t \text { and } \sum_{i=1}^{k} \gamma_{i} \int_{t_{i}}^{t_{i}+\varepsilon} Y(t) d t
$$

are independent for any choice of $\theta_{1}, \ldots, \theta_{k}$ and $\gamma_{1}, \ldots, \gamma_{k}$. Since all points $\left(t_{i}\right)$ are in the set $M$, dividing by $\varepsilon$ and letting $\varepsilon \rightarrow 0$ we conclude that

$$
\sum_{i=1}^{k} \theta_{i} X\left(t_{i}\right) \text { and } \sum_{i=1}^{k} \gamma_{i} Y\left(t_{i}\right)
$$

are independent for any choice of $\theta_{1}, \ldots, \theta_{k}$ and $\gamma_{1}, \ldots, \gamma_{k}$. By the Cramér-Wold device this implies that the vectors $\left(X\left(t_{1}\right), \ldots, X\left(t_{k}\right)\right)$ and

$\left(Y\left(t_{1}\right), \ldots, Y\left(t_{k}\right)\right)$ are independent.

\section{The bootstrap for the sample distance covariance}

We mentioned in Remark 3.3 that the limit distribution of $n T_{n, \beta}(X, Y)$ is not available. Theorem 3.1 states that the discretization $n T_{n, \beta}\left(X^{(p)}, Y^{(p)}\right)$ has the same asymptotic properties as $n T_{n, \beta}(X, Y)$ under suitable conditions on the smoothness of the sample paths, moment conditions and the growth rate of $p=p_{n} \rightarrow \infty$.

In this section we advocate the use of the bootstrap for approximating the distribution of $n T_{n, \beta}\left(X^{(p)}, Y^{(p)}\right)$. The bootstrap can be made to work for the degenerate $V$-statistic $T_{n, \beta}(X, Y)$ as shown in Arcones and Giné (1992). In this case, the naive bootstrap does not work and one has to modify the degenerate kernel. Since the $V$-statistic $T_{n, \beta}\left(X^{(p)}, Y^{(p)}\right)$ is degenerate for every fixed $p$ we face the problem of approximating the distribution of the latter statistic by its bootstrap version. We will show that this approximation works.

We will make use of a modification of Lemma 2.2 in Dehling and Mikosch (1994), which deals with $U$-statistics with a kernel defined on the Euclidean space. We work with a separable metric space $S$. For $m \geq 1$, let $h: S^{m} \mapsto \mathbb{R}$ be a symmetric function. Let $\left(X_{i}^{(1)}, X_{i}^{(2)}\right), i=1,2, \ldots$, be an $S \times S$-valued iid sequence with marginal laws $\mathcal{L}\left(X^{(1)}\right)=F$ and $\mathcal{L}\left(X^{(2)}\right)=G$, respectively. On the subset of probability measures on $S$,

$\Gamma_{2, h}=\left\{H: \mathbb{E}\left[h^{2}\left(Z_{1}, \ldots, Z_{m}\right)\right]<\infty\right.$ for iid $\left(Z_{i}\right)$ with common law $\left.H\right\}$, 
we define the semi-metric

$$
d_{2, h}(F, G)=\inf \left\{\left(\mathbb{E}\left[\left(h\left(X_{1}^{(1)}, \ldots, X_{m}^{(1)}\right)-h\left(X_{1}^{(2)}, \ldots, X_{m}^{(2)}\right)\right)^{2}\right]\right)^{1 / 2}\right\},
$$

where the infimum is taken over all random elements $\left(X_{1}^{(1)}, \ldots, X_{m}^{(1)}, X_{1}^{(2)}, \ldots, X_{m}^{(2)}\right)$ in $S^{2 m}$ such that $\left(X_{i}^{(1)}, X_{i}^{(2)}\right), i=1, \ldots, m$, are iid $S^{2}$-valued random elements, $X_{i}^{(1)}$ has law $F$ and $X_{i}^{(2)}$ has law $G$. The fact that $d_{2, h}$ is a semi-metric can be shown using similar arguments as in the proof of Lemma 8.1 in Bickel and Freedman (1981) that discusses the properties of the related Wasserstein metric $d_{2}$ on a subset of probability measures on $\mathbb{R}$, $\Gamma_{2}=\left\{H: \mathbb{E}_{H}\left[Z^{2}\right]<\infty\right\}$, defined by

$$
d_{2}(F, G)=\inf \left\{\left(\mathbb{E}\left[|A-B|^{2}\right]\right)^{1 / 2}: \mathcal{L}(A)=F, \mathcal{L}(B)=G\right\}
$$

Let $m \geq 2$ and choose $H \in \Gamma_{2, h}$. Define a function on $S \times S$ by

$$
\begin{aligned}
h_{2}(x, y ; H)= & \mathbb{E}\left[h\left(x, y, Z_{3}, \ldots, Z_{m}\right)\right]-\mathbb{E}\left[h\left(x, Z_{2}, \ldots, Z_{m}\right)\right] \\
& -\mathbb{E}\left[h\left(Z_{1}, y, Z_{3}, \ldots, Z_{m}\right)\right]+\mathbb{E}\left[h\left(Z_{1}, \ldots, Z_{m}\right)\right],
\end{aligned}
$$

where $\left(Z_{i}\right)$ are iid with common law $H$. The proof of the following result is completely analogous to that of Lemma 2.2 in Dehling and Mikosch (1994).

Lemma 5.1. Let $F, G$ be in $\Gamma_{2, h},\left(X_{j}^{(1)}\right)$ iid with common law $F$, and $\left(X_{j}^{(2)}\right)$ iid with common law $G$. Then for any $n \geq 1$,

$$
\begin{aligned}
& d_{2}\left(\mathcal{L}\left(\frac{1}{n} \sum_{1 \leq i \neq j \leq n} h_{2}\left(X_{i}^{(1)}, X_{j}^{(1)} ; F\right)\right), \mathcal{L}\left(\frac{1}{n} \sum_{1 \leq i \neq j \leq n} h_{2}\left(X_{i}^{(2)}, X_{j}^{(2)} ; G\right)\right)\right) \\
& \quad \leq 2^{5 / 2} d_{2, h}(F, G)
\end{aligned}
$$

For an $S$-valued iid sequence $\left(Z_{i}\right)$ with common law $F \in \Gamma_{2, h}$ and $n \geq 1$ we denote by $F_{n}$ the empirical law of $Z_{1}, \ldots, Z_{n}$. Consider an iid sequence $\left(Z_{n i}^{*}\right)$ with the law $F_{n}$, that is, given that law, independent of $\left(Z_{i}\right)$. The following result is analogous to Theorem 2.1 in Dehling and Mikosch (1994).

Corollary 5.2. Under the aforementioned conditions, and if also $\mathbb{E}\left[\left|h\left(Z_{i_{1}}, \ldots, Z_{i_{m}}\right)\right|^{2}\right]<\infty$ for all indices $1 \leq i_{1} \leq \ldots \leq i_{m} \leq m$, we have

$$
d_{2}\left(\mathcal{L}\left(\frac{1}{n} \sum_{1 \leq i \neq j \leq n} h_{2}\left(Z_{n i}^{*}, Z_{n j}^{*} ; F_{n}\right)\right), \mathcal{L}\left(\frac{1}{n} \sum_{1 \leq i \neq j \leq n} h_{2}\left(Z_{i}, Z_{j} ; F\right)\right)\right) \rightarrow 0
$$

for almost all realizations of $\left(Z_{i}\right)$.

Proof. By (5.2), it suffices to show that $d_{2, h}\left(F_{n}, F\right) \rightarrow 0$, almost surely. By Varadarajan's theorem (see Billingsley (1968), p.29) the empirical distribution $F_{n}$ converges weakly to the distribution $F$, for almost all realizations $\left(z_{i}\right)_{i \geq 1}$ of $\left(Z_{i}\right)_{i \geq 1}$. Thus, by Skorokhod's theorem, there exist a sequence of random variables $\left(Z_{n}^{*}\right)_{n \geq 1}$ such that $Z_{n}^{*}$ has distribution $F_{n}$, and an $F$-distributed random 
variable $\tilde{Z}$ such that $Z_{n}^{*} \rightarrow \tilde{Z}$ almost surely. We now take $m$ iid copies of the pair $\left(Z_{n}^{*}, \tilde{Z}\right)$, which we denote by $\left(Z_{n 1}^{*}, \tilde{Z}_{1}\right), \ldots,\left(Z_{n m}, \tilde{Z}_{m}\right)$. Then

$$
\left(Z_{n 1}^{*}, \ldots, Z_{n m}^{*}\right) \rightarrow\left(\tilde{Z}_{1}, \ldots, \tilde{Z}_{m}\right), \text { almost surely. }
$$

Moreover, by definition of $d_{2, h}$, we have

$$
d_{2, h}\left(F_{n}, F\right) \leq\left(\mathbb{E}\left[\left(h\left(Z_{n 1}^{*}, \ldots, Z_{n m}^{*}\right)-h\left(\tilde{Z}_{1}, \ldots, \tilde{Z}_{m}\right)\right)^{2}\right]\right)^{1 / 2} .
$$

It suffices to show that the right-hand side converges to 0 as $n \rightarrow \infty$. For any $\epsilon>0$, we can find a bounded continuous function $g: S^{m} \rightarrow \mathbb{R}$ such that

$$
\mathbb{E}\left[\left(h\left(\tilde{Z}_{1}, \ldots, \tilde{Z}_{m}\right)-g\left(\tilde{Z}_{1}, \ldots, \tilde{Z}_{m}\right)\right)^{2}\right] \leq \epsilon .
$$

By Lebesgue's dominated convergence theorem, we obtain

$$
\mathbb{E}\left[\left(g\left(Z_{n, 1}^{*}, \ldots, Z_{n, m}^{*}\right)-g\left(\tilde{Z}_{1}, \ldots, \tilde{Z}_{m}\right)\right)^{2}\right] \rightarrow 0 .
$$

The strong law of large numbers for $U$-statistics implies that

$$
\begin{aligned}
\mathbb{E} & {\left[\left(h\left(Z_{n, 1}^{*}, \ldots, Z_{n, m}^{*}\right)-g\left(Z_{n, 1}^{*}, \ldots, Z_{n, m}^{*}\right)\right)^{2}\right] } \\
& =\frac{1}{n^{m}} \sum_{1 \leq i_{1}, \ldots, i_{m} \leq n}\left(h\left(z_{i_{1}}, \ldots, z_{i_{m}}\right)-g\left(z_{i_{1}}, \ldots, z_{i_{m}}\right)\right)^{2} \\
& \rightarrow \mathbb{E}\left(h\left(Z_{1}, \ldots, Z_{m}\right)-g\left(Z_{1}, \ldots, Z_{m}\right)\right)^{2} \leq \epsilon
\end{aligned}
$$

This finishes the proof.

In what follows, $\left(Z_{i}\right)$ will stand for the iid sequence of the pairs $\left(X_{i}, Y_{i}\right), i=$ $1,2, \ldots$, used in the previous sections for defining the quantities $T_{n, \beta}(X, Y)$. Correspondingly, we write $\left(Z_{i}^{(p)}\right)$ for the sequence of the discretizations $\left(X_{i}^{(p)}, Y_{i}^{(p)}\right)$, $i=1,2, \ldots$, with generic element $Z^{(p)}$. For the ease of presentation we focus on the case $\beta=1$ and suppress $\beta$ in the notation. We consider only the case when $X, Y$ have finite second moments. A generic element $Z=(X, Y)$ has trajectory $(x, y)$ assuming values in a function space $S$ where $x, y$ are defined on $[0,1]$ and are Riemann square-integrable.

Under the hypothesis that $X, Y$ are independent, $T_{n}(X, Y)$ has representation as a $V$-statistic of order 4 with a 1-degenerate symmetric kernel $h_{4}=$ $h\left(x_{1}, x_{2}, x_{3}, x_{4}\right)$; see Appendix A, where we also show that, when scaled by $n$, the limits of $T_{n}(X, Y)$ and the corresponding normalized $U$-statistic (which is obtained by ignoring all summands $h\left(Z_{i_{1}}, Z_{i_{2}}, Z_{i_{3}}, Z_{i_{4}}\right)$ with the property $i_{j}=i_{k}$ for $j \neq k$ ) differ by an additive constant. Applying the Hoeffding decomposition to this $U$-statistic, the limiting distribution of $n T_{n}(X, Y)$ coincides, up to a scale change, with the limiting distribution of the following normalized $U$-statistic:

$$
U_{n}(Z)=\frac{1}{n} \sum_{1 \leq i \neq j \leq n} h_{2}\left(Z_{i}, Z_{j} ; F_{Z}\right)
$$


where $F_{Z}=F_{X} \times F_{Y}$ and $h_{2}$ is defined in (5.1). Arcones and Giné (1992) proved that the correct bootstrap version of $n T_{n}(X, Y)$ is

$$
U_{n}\left(Z^{*}\right)=\frac{1}{n} \sum_{1 \leq i \neq j \leq n} h_{2}\left(Z_{n i}^{*}, Z_{n j}^{*} ; F_{n, Z}\right),
$$

where $F_{n, Z}$ is the empirical distribution of the iid sample $Z_{1}, \ldots, Z_{n}$. The fact that the limiting distributions of $U_{n}(Z)$ and $U_{n}\left(Z^{*}\right)$ coincide follows from Corollary 5.2.

Our program for the remainder of this section is to show that we are allowed to replace $Z=(X, Y)$ by the corresponding discretizations $Z^{(p)}=\left(X^{(p)}, Y^{(p)}\right)$ in the aforementioned $U$ - and $V$-statistics, i.e., we will show that suitable bootstrap versions of $n T_{n, \beta}(X, Y)$ and $n T_{n, \beta}\left(X^{(p)}, Y^{(p)}\right)$ have the same limiting distribution. We start by showing that $U_{n}(Z)$ and $U_{n}\left(Z^{(p)}\right)$ are close in the sense of the $d_{2}$-metric.

Lemma 5.3. Assume the following conditions:

1. $X, Y$ are independent and have finite second moments.

2. Condition (A1) holds.

3. $\delta_{n} \rightarrow 0$ as $n \rightarrow \infty$.

Then $d_{2}\left(\mathcal{L}\left(U_{n}(Z)\right) ; \mathcal{L}\left(U_{n}\left(Z^{(p)}\right)\right)\right) \leq c \delta_{n}^{\left(\gamma_{X} \wedge \gamma_{Y}\right) / 2} \rightarrow 0$.

Proof. By (5.2), with $h$ given by (A.1), we have

$$
\begin{aligned}
& d_{2}\left(\mathcal{L}\left(U_{n}(Z)\right) ; \mathcal{L}\left(U_{n}\left(Z^{(p)}\right)\right)\right. \\
& \quad \leq c\left\{\mathbb{E}\left[\left(h\left(Z_{1}, \ldots, Z_{4}\right)-h\left(Z_{1}^{(p)}, \ldots, Z_{4}^{(p)}\right)\right)^{2}\right]\right\}^{1 / 2} \\
& \quad \leq c\left\{\mathbb{E}\left[\left(f\left(Z_{1}, \ldots, Z_{4}\right)-f\left(Z_{1}^{(p)}, \ldots, Z_{4}^{(p)}\right)\right)^{2}\right]\right\}^{1 / 2} \\
& \quad \leq c\left(\mathbb{E} I_{1}^{2}+\mathbb{E} I_{2}^{2}+\mathbb{E} I_{3}^{2}\right)^{1 / 2}
\end{aligned}
$$

where

$$
\begin{aligned}
I_{1} & =\left\|X_{1}-X_{2}\right\|_{2}\left\|Y_{1}-Y_{2}\right\|_{2}-\left\|X_{1}^{(p)}-X_{2}^{(p)}\right\|_{2}\left\|Y_{1}^{(p)}-Y_{2}^{(p)}\right\|_{2}, \\
I_{2} & =\left\|X_{1}-X_{2}\right\|_{2}\left\|Y_{3}-Y_{4}\right\|_{2}-\left\|X_{1}^{(p)}-X_{2}^{(p)}\right\|_{2}\left\|Y_{3}^{(p)}-Y_{4}^{(p)}\right\|_{2}, \\
I_{3} & =\left\|X_{1}-X_{2}\right\|_{2}\left\|Y_{1}-Y_{3}\right\|_{2}-\left\|X_{1}^{(p)}-X_{2}^{(p)}\right\|_{2}\left\|Y_{1}^{(p)}-Y_{3}^{(p)}\right\|_{2} .
\end{aligned}
$$

The second moments are estimated as in Proposition 7.1 below. We have by (7.4),

$$
\mathbb{E}\left[\left(\left\|X_{1}-X_{2}\right\|_{2}-\left\|X_{1}^{(p)}-X_{2}^{(p)}\right\|_{2}\right)^{2}\left\|Y_{1}-Y_{2}\right\|_{2}^{2}\right] \leq c \delta_{n}^{\gamma_{X}}
$$

and

$$
\mathbb{E}\left[\left\|X_{1}^{(p)}-X_{2}^{(p)}\right\|_{2}^{2}\left(\left\|Y_{1}-Y_{2}\right\|_{2}-\left\|Y_{1}^{(p)}-Y_{2}^{(p)}\right\|_{2}\right)^{2}\right] \leq c \delta_{n}^{\gamma_{Y}} .
$$

That is, $\mathbb{E}\left[I_{1}^{2}\right] \leq c \delta_{n}^{\gamma_{X} \wedge \gamma_{Y}}$. The second moments of $I_{2}, I_{3}$ can be bounded by the same quantities. 
Our next goal is to show that, under appropriate assumptions, the difference between the laws of $U_{n}\left(Z^{*}\right)$ and $U_{n}\left(Z^{(p) *}\right)$ asymptotically vanishes.

Lemma 5.4. Consider the following conditions:

1. $X, Y$ are independent and have finite second moments.

2a. Condition (A1) holds.

2b. $\mathbb{E}\left[|X(t)-X(s)|^{4}\right] \leq c|t-s|^{\widetilde{\gamma}_{X}}$ and $\mathbb{E}\left[|Y(t)-Y(s)|^{4}\right] \leq c|t-s|^{\widetilde{\gamma}_{Y}}$ hold.

3a. $\sum_{n=1}^{\infty} \delta_{n}^{\gamma_{X} \wedge \gamma_{Y}<\infty}$.

3b. $\sum_{n=1}^{\infty}\left(\delta_{n}^{2\left(\gamma_{X} \wedge \gamma_{Y}\right)}+n^{-1} \delta_{n}^{\left.\widetilde{\gamma}_{X} \wedge \widetilde{\gamma}_{Y}\right)<\infty .}\right.$

If either $1,2 \mathrm{a}$, 3a or $1,2 \mathrm{a}, 2 \mathrm{~b}, 3 \mathrm{~b}$ hold then $d_{2}\left(\mathcal{L}\left(U_{n}\left(Z^{*}\right)\right), \mathcal{L}\left(U_{n}\left(Z^{(p) *}\right)\right)\right) \rightarrow 0$, for a.e. realization of $\left(Z_{i}\right)$.

Proof. With $h$ given by $\left(\right.$ A.1), by Lemma 5.1 it is enough to prove that $d_{2, h}\left(\mathcal{L}\left(Z^{*}\right), \mathcal{L}\left(Z^{(p) *}\right)\right) \rightarrow$ 0 for a.e. realization of $\left(Z_{i}\right)$. We have

$$
\begin{aligned}
& d_{2, h}(n):=d_{2, h}\left(\mathcal{L}\left(Z^{*}\right), \mathcal{L}\left(Z^{(p) *}\right)\right) \\
& \quad \leq \quad\left(\mathbb{E}_{F_{n}}\left[\left(h\left(Z_{1}^{*}, Z_{2}^{*}, Z_{3}^{*}, Z_{4}^{*}\right)-h\left(Z_{1}^{(p) *}, Z_{2}^{(p) *}, Z_{3}^{(p) *}, Z_{4}^{(p) *}\right)\right)^{2}\right]\right)^{1 / 2} \\
& \quad=\frac{1}{n^{2}}\left(\sum_{1 \leq i_{1}, i_{2}, i_{3}, i_{4} \leq n}\left(h\left(Z_{i_{1}}, Z_{i_{2}}, Z_{i_{3}}, Z_{i_{4}}\right)-h\left(Z_{i_{1}}^{(p)}, Z_{i_{2}}^{(p)}, Z_{i_{3}}^{(p)}, Z_{i_{4}}^{(p)}\right)\right)^{2}\right)^{1 / 2} \\
& \quad \leq \frac{1}{n^{2}}\left(\sum_{1 \leq i_{1}, i_{2}, i_{3}, i_{4} \leq n}\left(f\left(Z_{i_{1}}, Z_{i_{2}}, Z_{i_{3}}, Z_{i_{4}}\right)-f\left(Z_{i_{1}}^{(p)}, Z_{i_{2}}^{(p)}, Z_{i_{3}}^{(p)}, Z_{i_{4}}^{(p)}\right)\right)^{2}\right)^{1 / 2} .
\end{aligned}
$$

We first show that the right-hand side converges to zero under the assumption that 1, 2a, and 3a hold. Using (A1), we obtain

$$
\begin{aligned}
& \mathbb{E}\left[d_{2, h}\left(\mathcal{L}\left(Z^{*}\right), \mathcal{L}\left(Z^{(p) *}\right)\right)\right]^{2} \\
& \leq \sum_{1 \leq j_{1}, j_{2}, j_{3}, j_{4} \leq 4} \mathbb{E}\left[\left(f\left(Z_{j_{1}}, \ldots, Z_{j_{4}}\right)-f\left(Z_{j_{1}}^{(p)}, \ldots, Z_{j_{4}}^{(p)}\right)\right)^{2}\right] \leq c \delta_{n}^{\gamma_{X} \wedge \gamma_{Y}} .
\end{aligned}
$$

Thus, if $\sum_{n} \delta_{n}^{\gamma_{X} \wedge \gamma_{Y}}<\infty$ applications of Markov's inequality and the BorelCantelli lemma yield that $d_{2, h}\left(\mathcal{L}\left(Z^{*}\right), \mathcal{L}\left(Z^{(p) *}\right)\right) \rightarrow 0$ a.s. as $n \rightarrow \infty$.

Now assume that $1,2 \mathrm{a}, 2 \mathrm{~b}$ and $3 \mathrm{~b}$ hold. Using standard calculations for $U$-statistics, we have

$$
\begin{aligned}
\operatorname{var}\left(d_{2, h}^{2}(n)\right) \leq & c \sum_{1 \leq j_{1}, j_{2}, j_{3}, j_{4} \leq 4}\left[n^{-1} \operatorname{var}\left(\left(h\left(Z_{j_{1}}, \ldots, Z_{j_{4}}\right)-h\left(Z_{j_{1}}^{(p)}, \ldots, Z_{j_{4}}^{(p)}\right)\right)^{2}\right)\right. \\
& \left.+\left(\mathbb{E}\left[\left(h\left(Z_{j_{1}}, \ldots, Z_{j_{4}}\right)-h\left(Z_{j_{1}}^{(p)}, \ldots, Z_{j_{4}}^{(p)}\right)\right)^{2}\right]\right)^{2}\right]=J_{1}+J_{2} .
\end{aligned}
$$

We have $J_{2}=O\left(\delta_{n}^{2\left(\gamma_{X} \wedge \gamma_{Y}\right)}\right)$. We can handle $J_{1}$ similarly to the proof of Lemma 5.3. For example,

$$
\mathbb{E}\left[\left\|X_{1}-X_{1}^{(p)}\right\|_{2}^{4}\right]=\mathbb{E}\left[\left(\int_{0}^{1}\left(X(u)-X^{(p)}(u)\right)^{2} d u\right)^{2}\right]
$$




$$
\leq c \int_{0}^{1} \mathbb{E}\left[\left(X(u)-X^{(p)}(u)\right)^{4}\right] d u \leq c \delta_{n}^{\widetilde{\gamma}_{X}}
$$

Now $d_{2, h}(n) \stackrel{\text { a.s. }}{\rightarrow} 0$ as $n \rightarrow \infty$ follows by an application of Markov's inequality of order 2 , the Borel-Cantelli lemma and since $\sum_{n}\left(n^{-1} \delta_{n}^{\widetilde{\gamma}_{X} \wedge \widetilde{\gamma}_{Y}}+\delta_{n}^{2\left(\gamma_{X} \wedge \gamma_{Y}\right)}\right)<\infty$. We omit further details.

Combining the previous arguments, a natural bootstrap version of the degenerate $V$-statistic $n T_{n}\left(X^{(p)}, Y^{(p)}\right)$ is given by $U_{n}\left(Z^{(p) *}\right)$.

Proposition 5.5. Assume the conditions of Lemma 5.4. Then

$$
d_{2}\left(\mathcal{L}\left(U_{n}(Z)\right), \mathcal{L}\left(U_{n}\left(Z^{(p) *}\right)\right)\right) \rightarrow 0
$$

for a.e. realization of $\left(Z_{i}\right)$.

For an application of the bootstrapped sample distance correlation $n R_{n}\left(X^{(p)}, Y^{(p)}\right)$ we still miss one step in the derivation of the bootstrap consistency: we also need to prove that the denominator quantities converge a.s.

$$
T_{n}\left(X^{(p)}, X^{(p)}\right) \stackrel{\text { a.s. }}{\rightarrow} T(X, X) \quad \text { and } \quad T_{n}\left(Y^{(p)}, Y^{(p)}\right) \stackrel{\text { a.s. }}{\rightarrow} T(Y, Y), \quad n \rightarrow \infty .
$$

In Lemma B.1 Appendix B we provide sufficient conditions for this to hold.

\section{Simulations}

In this section we illustrate the theoretical results in a small simulation study. Throughout we choose $\beta=1$ and suppress the dependence on $\beta$ in the notation.

We start with identically distributed fractional Brownian motions (fBM) $X, Y$ on $[0,1]$ with Hurst coefficient $H$ and correlation $\rho$ where the dependence between $X$ and $Y$ is given by the covariance function

$$
\operatorname{cov}(X(s), Y(t))=\frac{\rho}{2}\left\{|s|^{2 H}+|t|^{2 H}-|t-s|^{2 H}\right\}, s, t \in[0,1] .
$$

If $X=Y$ we also set $\rho=1$. Note that, for $H=1 / 2$, the right-hand side collapses into $\rho(s \wedge t)$, corresponding to Brownian motions $X, Y$. The top graph in Figure 1 nicely illustrates the consistency of the sample correlation $R_{n}\left(X^{(p)}, Y^{(p)}\right)$ for independent $X$ and $Y(\rho=0)$. In the top row we fix $p=100$ and increase $n$ from 100 to 400 , and we choose $H=1 / 4, H=1 / 2(\mathrm{BM})$ and $H=3 / 4$. Apparently, we can see the influence of the smoothness of the sample paths: the larger $H$ the larger $\gamma_{X}=\gamma_{Y}=2 H$ (see Example 2.3), the smoother the sample paths and the closer $R_{n}\left(X^{(p)}, Y^{(p)}\right)$ to zero; see also the upper bounds in Proposition 7.1. In the bottom row we show $R_{n}\left(X^{(p)}, Y^{(p)}\right)$ for dependent $X$ and $Y$ with $\rho=0.5$. We again choose $H=1 / 4, H=1 / 2(\mathrm{BM})$ and $H=3 / 4$, fix $p=100$ and increase $n$ from 100 to 300. In the bottom graphs the sample distance correlation converges to some positive constants; we see a clear difference between the independent and dependent cases. 

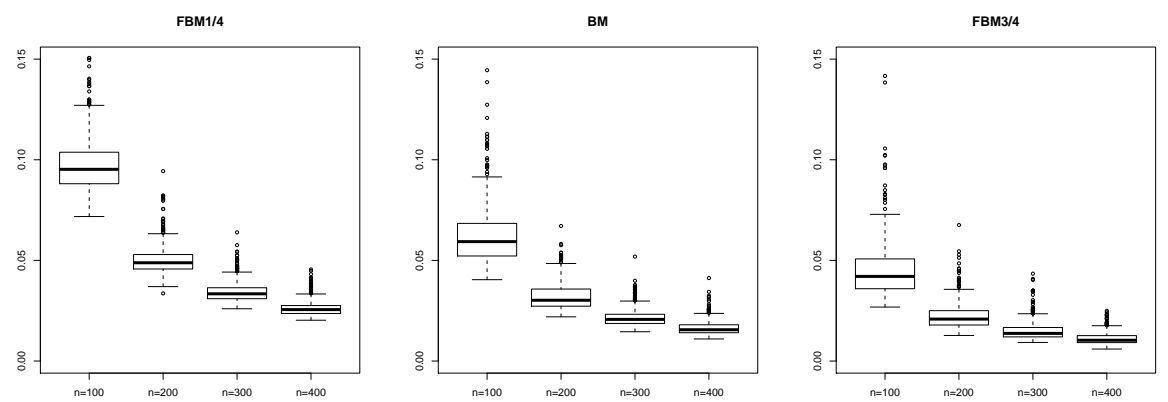

FBM $1 / 4$ dependent
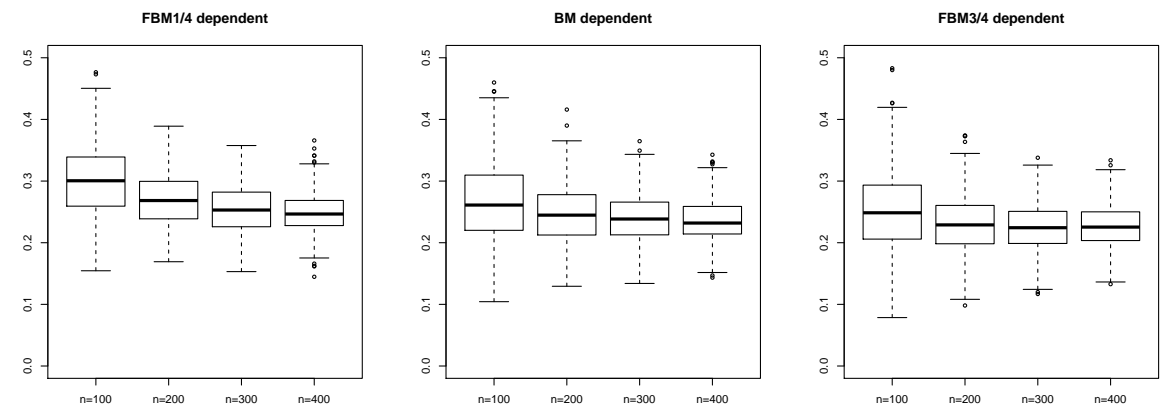

FIG 1. Boxplots for $R_{n}\left(X^{(p)}, Y^{(p)}\right)$ simulated fBMs $X, Y$ with $H=1 / 4,1 / 2,3 / 4$ (from left to right), $p=100$ and increasing sample sizes $n$. Top: iid $f B M s X, Y$. Each boxplot is based on 500 replications. Bottom: identically distributed $f B M s X, Y$ with correlation $\rho=0.5$. Each boxplot is based on 300 replications.
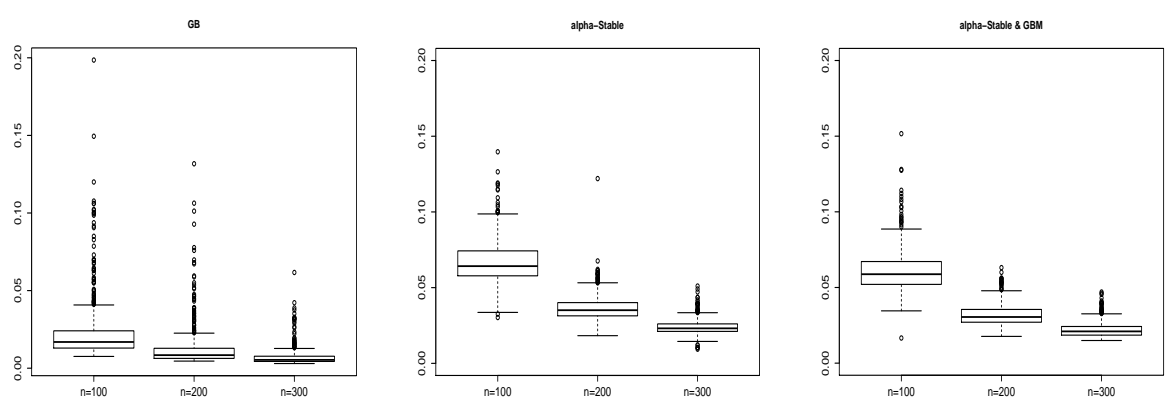

Fig 2. Boxplots for $R_{n}\left(X^{(p)}, Y^{(p)}\right)$ for simulated independent non-Gaussian processes $X, Y$, $p=100$ and increasing sample size $n$. Each boxplot is based on 500 replications. Left: iid geometric BMs $X, Y$. Middle: iid $\alpha$-stable Lévy motions $X, Y$. Right: independent geometric $B M X$ and $\alpha$-stable Lévy motion $Y$. 

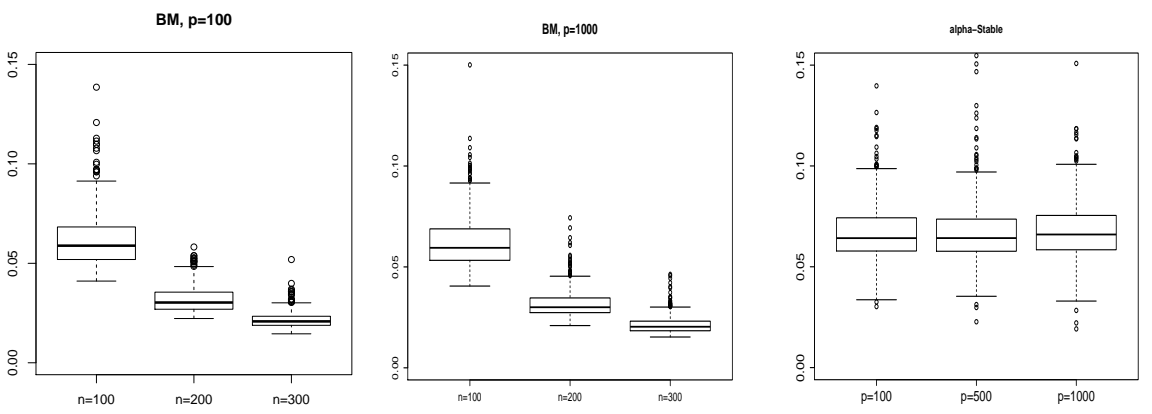

Fig 3. Boxplots for $R_{n}\left(X^{(p)}, Y^{(p)}\right)$ for different $p$. Left and middle: $X, Y$ are iid $B M s$. For each $p=100$ (left) and $p=1000$ (middle) we take three distinct sample sizes $n=$ 100,200,300. The boxplots are based on 300 replications. Right: X, Y are iid $\alpha$-stable Lévy motions, $n=100$ is fixed while $p=100,500,1000$. The boxplots are based on 500 replications.
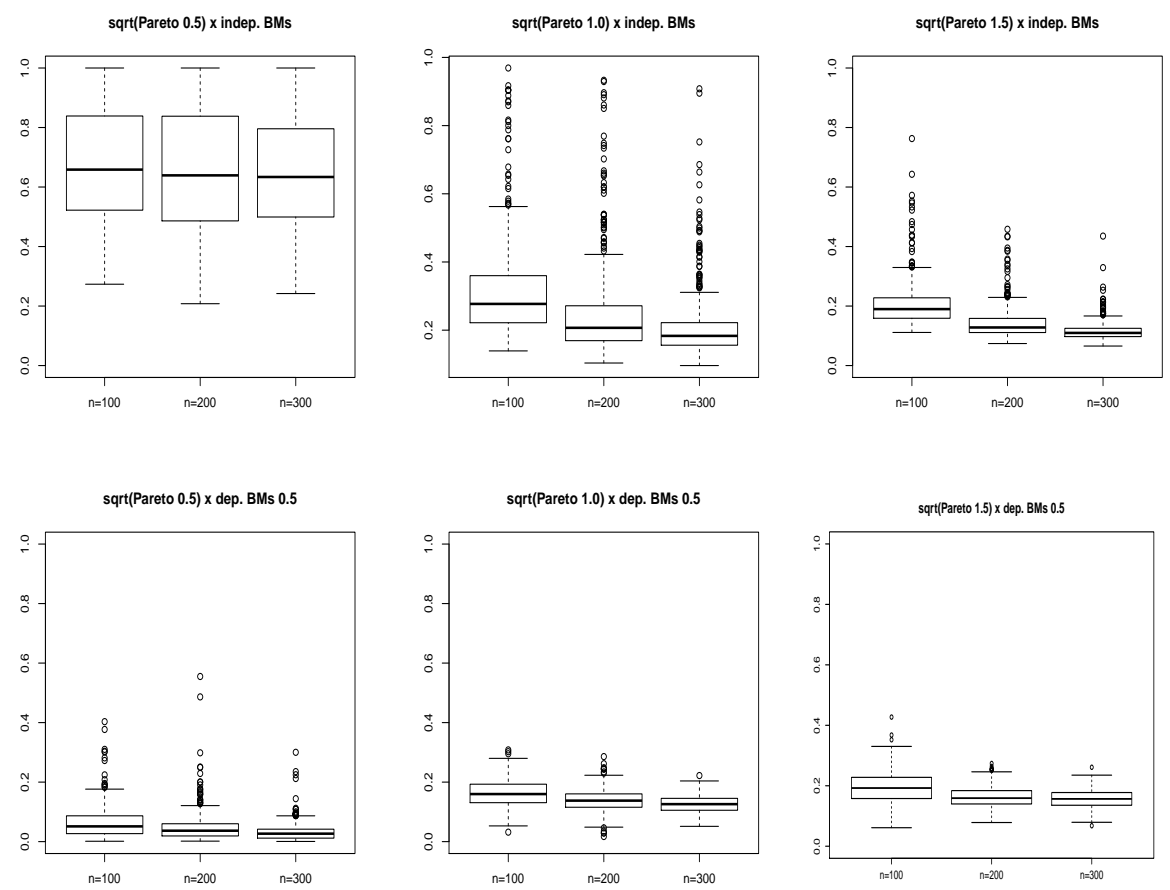

FIG 4. Boxplots of $R_{n}\left(X^{(p)}, Y^{(p)}\right)$ for dependent heavy-tail cases. Top: $(X, Y)=A^{1 / 2}\left(B_{1}, B_{2}\right)$ for a Pareto $(\alpha)$ variable $A$ independent of iid Brownian motions $\left(B_{1}, B_{2}\right)$. Bottom: $(X, Y)=$ $\left(A_{1}^{1 / 2} B_{1}, A_{2}^{1 / 2} B_{2}\right)$ for iid copies $A_{1}, A_{2}$ independent of the Brownian motions $B_{1}, B_{2}$ with correlation $\rho=0.5$. From left to right: $\alpha=0.5,1.0,1.5$. Sample sizes $n=100,200,300$, $p=100$, and each plot is based on 500 replications. 

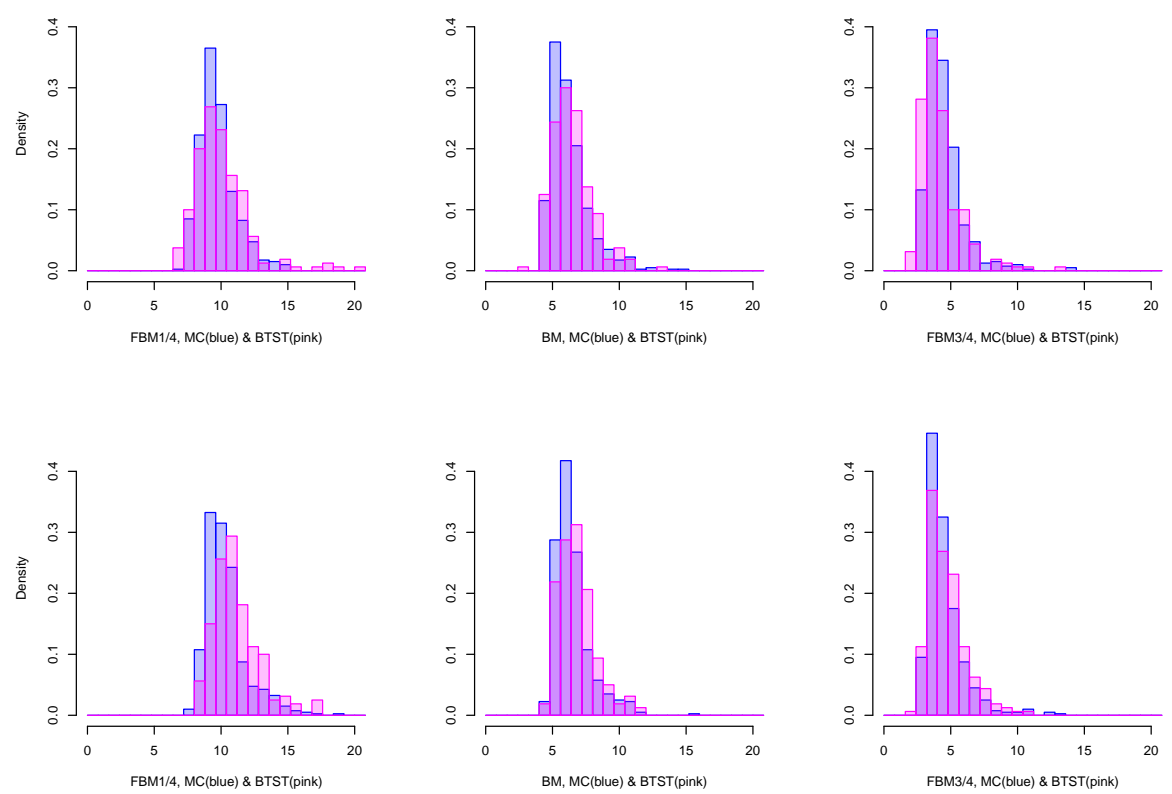

FIG 5. Comparison of histograms for $n R_{n}\left(X^{(p)}, Y^{(p)}\right)$ based on Monte Carlo simulation (blue) and bootstrap (pink) for iid fBMs $X, Y$ with $H=1 / 4, H=1 / 2, H=3 / 4$ (from left to right). The sample size is $n=100$ (top) and $n=300$ (bottom) and $p=100$. The histograms of $n R_{n}\left(X^{(p)}, Y^{(p)}\right)$ and the bootstrap version are based on 500 and 200 replications, respectively.

In Figure 2 we illustrate the performance of the sample distance correlation $R_{n}\left(X^{(p)}, Y^{(p)}\right.$ ) when $X$ and $Y$ are independent (possibly with distinct distributions) non-Gaussian processes. We treat three cases, including heavy-tailed processes: $X, Y$ are iid geometric BMs (left), $X, Y$ are iid $\alpha$-stable Lévy motions (middle), $X$ is a geometric BM and $Y$ an $\alpha$-stable Lévy motion (right). For geometric BM we choose the parametrization

$$
X(t)=\exp \left(\left(1-0.7^{2} / 2\right) t+0.7 B(t), \quad t \in[0,1],\right.
$$

where $\mu=1$ (drift), $\sigma=0.7$ (volatility) and $B$ is standard BM. The parameters of the $\alpha$-stable Lévy motions are $(\alpha, \beta, \mu, \sigma)=(1.8,0.3,0,1)$; cf. (Samorodnitsky and Taqqu, 1994, Ex. 3.1.3). We fix $p=100$ and increase $n$ from 100 to 300. Also in these non-Gaussian settings the boxplots nicely illustrate consistency of $R_{n}\left(X^{(p)}, Y^{(p)}\right)$ even in the heavy-tailed $\alpha$-stable case.

In Figure 3 we study the influence of the size of $p$ on the sample distance correlation for a given $n$. We choose $p=100$ (left) and $p=1000$ (middle) while $X, Y$ are independent BMs: there is hardly any difference between the left and middle graphs for a given $n$. In the right graph we choose iid $\alpha$-stable Lévy 
motions $X, Y$ with the same parameters as before. We increased $p$ from 100 to 1000 and fix $n=100$. Again, one can hardly see any difference between the boxplots. These observations are not surprising - in view of the definition of the distance correlation and the independence of $X^{(p)}$ and $Y^{(p)}$ for any $p$. However, it is perhaps unexpected that $n$ and $p$ may have similar size and still provide good approximations to zero. In Figure 5 we visualize how the bootstrap works for the normalized sample distance correlations $n R_{n}\left(X^{(p)}, Y^{(p)}\right)$ for iid fBMs $X, Y$. We show histograms based on 500 replications of $n R_{n}\left(X^{(p)}, Y^{(p)}\right)$ and compare with the histograms based on 200 replications of the bootstrap version generated from a single sample. We see that the distributions of $n R_{n}\left(X^{(p)}, Y^{(p)}\right)$ and its bootstrap version are close to each other and get more concentrated.

We also examine some dependent heavy-tailed cases. We have chosen two simple stochastic process models for $X, Y$ where we can control the tails and the dependence. First, we consider iid standard BMs $B_{1}, B_{2}$ which are subject to a joint heavy-tailed shock, $(X, Y)=A^{1 / 2}\left(B_{1}, B_{2}\right)$, where $A$ is a $\operatorname{Pareto}(\alpha)$ variable for some $\alpha>0$ with density $f_{\alpha}(x)=\alpha(1+x)^{-(\alpha+1)}, x>0$. We also assume that $A$ and $\left(B_{1}, B_{2}\right)$ are independent. Notice that $A^{1 / 2}$ does not have a $2 \alpha$ th moment. Second, we consider $(X, Y)=\left(A_{1}^{1 / 2} B_{1}, A_{2}^{1 / 2} B_{2}\right)$ where $A_{1}, A_{2}$ are iid copies of $A$ with density $f_{\alpha}$, independent of $\left(B_{1}, B_{2}\right)$ while $B_{1}$ and $B_{2}$ are dependent BMs with correlation $\rho=0.5$. We have chosen $2 \alpha=1,2,3$. In the case $\alpha=0.5$ the theoretical results of this paper about consistency of $T_{n}\left(X^{(p)}, Y^{(p)}\right)$ do not apply since $\mathbb{E}\left[\|X\|_{2}+\|Y\|_{2}\right]=\infty$ while in the cases $\alpha=1,1.5, T_{n}\left(X^{(p)}, Y^{(p)}\right) \stackrel{\mathbb{P}}{\rightarrow} T(X, Y)>0$.

The first/second model is examined in the top/bottom graphs of Figure 4, respectively. In the cases $\alpha=1,1.5$ the centers of the boxplots seem to stabilize with increasing sample size, pointing at the consistency of $R_{n}\left(X^{(p)}, Y^{(p)}\right)$. In the top graphs (first model) we observe that the distributions of $R_{n}\left(X^{(p)}, Y^{(p)}\right)$ have a rather wide range while the bottom boxplots (second model) are less spread and their center is much below those of the first model. Moreover, in the $\alpha=0.5$ case the plot is close to zero. It could be taken as a false indication of independence between $X$ and $Y$. We do not have a full explanation for the phenomena observed in Figure 4; in both heavy-tailed dependent models our assumptions for the existence of non-degenerate weak limits are not satisfied due to the lack of moments.

\section{Proof of Theorem 3.1}

We prove the theorem by a series of auxiliary results.

Proposition 7.1. Assume the conditions 1.-4. of Theorem 3.1.

1. If also (A1) holds then there is $c$ such that for any $n \geq 1$,

$$
\mathbb{E}\left[\left|T_{n, \beta}\left(X^{(p)}, Y^{(p)}\right)-T_{n, \beta}(X, Y)\right|\right] \leq c \delta_{n}^{\left(\gamma_{X} \wedge \gamma_{Y}\right)(\beta \wedge 1) / 2} .
$$

2. If also (B1),(B2) hold then there is $c$ such that

$$
\mathbb{E}\left[\left|T_{n, \beta}\left(X^{(p)}, Y^{(p)}\right)-T_{n, \beta}(X, Y)\right|\right] \leq c\left(p \delta_{n}^{\left(\beta / 2+\gamma_{X} \wedge \gamma_{Y}\right)}\right)^{(\beta \wedge 1) / \beta} .
$$

imsart-generic ver. 2014/10/16 file: dmms12018main_2ndsubmissionArxiv.tex date: November 30, 2018 
Proof. We start with the decomposition

$$
T_{n, \beta}\left(X^{(p)}, Y^{(p)}\right)-T_{n, \beta}(X, Y)=I_{1}+I_{2}-2 I_{3},
$$

where

$$
\begin{aligned}
I_{1}= & \frac{1}{n^{2}} \sum_{k, l=1}^{n}\left(\left\|X_{k}^{(p)}-X_{l}^{(p)}\right\|_{2}^{\beta}\left\|Y_{k}^{(p)}-Y_{l}^{(p)}\right\|_{2}^{\beta}-\left\|X_{k}-X_{l}\right\|_{2}^{\beta}\left\|Y_{l}-Y_{k}\right\|_{2}^{\beta}\right), \\
I_{2}= & \frac{1}{n^{4}} \sum_{k, l=1}^{n}\left\|X_{k}^{(p)}-X_{l}^{(p)}\right\|_{2}^{\beta} \sum_{k, l=1}^{n}\left\|Y_{k}^{(p)}-Y_{l}^{(p)}\right\|_{2}^{\beta} \\
& \quad-\frac{1}{n^{4}} \sum_{k, l=1}^{n}\left\|X_{k}-X_{l}\right\|_{2}^{\beta} \sum_{k, l=1}^{n}\left\|Y_{k}-Y_{l}\right\|_{2}^{\beta}, \\
I_{3}= & \frac{1}{n^{3}} \sum_{k, l, m=1}^{n}\left\|X_{k}^{(p)}-X_{l}^{(p)}\right\|_{2}^{\beta}\left\|Y_{k}^{(p)}-Y_{m}^{(p)}\right\|_{2}^{\beta} \\
& \quad-\frac{1}{n^{3}} \sum_{k, l, m=1}^{n}\left\|X_{k}-X_{l}\right\|_{2}^{\beta}\left\|Y_{k}-Y_{m}\right\|_{2}^{\beta} .
\end{aligned}
$$

We will find bounds for the absolute values of the expectations of these quantities. From now on, $c$ denotes any positive constants whose values are not of interest.

First assume that $(X, Y)$ have finite second moment. Observe that

$$
\begin{aligned}
\left|I_{1}\right| \leq & \frac{1}{n^{2}} \sum_{k, l=1}^{n}\left|\left\|X_{k}^{(p)}-X_{l}^{(p)}\right\|_{2}^{\beta}-\left\|X_{k}-X_{l}\right\|_{2}^{\beta}\right|\left\|Y_{k}^{(p)}-Y_{l}^{(p)}\right\|_{2}^{\beta} \\
& +\frac{1}{n^{2}} \sum_{k, l=1}^{n}\left|\left\|Y_{k}^{(p)}-Y_{l}^{(p)}\right\|_{2}^{\beta}-\left\|Y_{k}-Y_{l}\right\|_{2}^{\beta}\right|\left\|X_{k}-X_{l}\right\|_{2}^{\beta}=: I_{11}+I_{12} .
\end{aligned}
$$

By a symmetry argument, interchanging the roles of $X$ and $Y$, it suffices to consider $I_{11}$. Using the independence of $X$ and $Y$, we have

$$
\mathbb{E}\left[I_{11}\right] \leq \mathbb{E}\left[\left|\left\|X_{1}^{(p)}-X_{2}^{(p)}\right\|_{2}^{\beta}-\left\|X_{1}-X_{2}\right\|_{2}^{\beta}\right|\right] \mathbb{E}\left[\left\|Y_{1}^{(p)}-Y_{2}^{(p)}\right\|_{2}^{\beta}\right] .
$$

By Lyapunov's inequality,

$$
\begin{aligned}
\mathbb{E}\left[\left\|Y_{1}^{(p)}-Y_{2}^{(p)}\right\|_{2}^{\beta}\right] & \leq\left(\mathbb{E}\left[\left\|Y_{1}^{(p)}-Y_{2}^{(p)}\right\|_{2}^{2}\right]\right)^{\beta / 2} \\
& \leq c\left(\int_{0}^{1} \operatorname{var}\left(Y^{(p)}(t)\right) d t\right)^{\beta / 2}<\infty .
\end{aligned}
$$

Assume $0<\beta \leq 1$. Then, by concavity and Jensen's inequality,

$$
\begin{aligned}
& \mathbb{E}\left[\left|\left\|X_{1}^{(p)}-X_{2}^{(p)}\right\|_{2}^{\beta}-\left\|X_{1}-X_{2}\right\|_{2}^{\beta}\right|\right] \\
& \quad \leq \mathbb{E}\left[\left\|\left(X_{1}^{(p)}-X_{2}^{(p)}\right)-\left(X_{1}-X_{2}\right)\right\|_{2}^{\beta}\right]
\end{aligned}
$$




$$
\begin{aligned}
& =\mathbb{E}\left[\left(\sum_{i=1}^{p} \int_{\Delta_{i}}\left(\Delta X_{1}\left(t, t_{i}\right]-\Delta X_{2}\left(t, t_{i}\right]\right)^{2} d t\right)^{\beta / 2}\right] \\
& \leq\left(\sum_{i=1}^{p} \int_{\Delta_{i}} \operatorname{var}\left(\Delta X_{1}\left(t, t_{i}\right]-\Delta X_{2}\left(t, t_{i}\right]\right) d t\right)^{\beta / 2} \\
& =\left(\sum_{i=1}^{p} \int_{\Delta_{i}}\left(\operatorname{var}\left(\Delta X_{1}\left(t, t_{i}\right]\right)+\operatorname{var}\left(\Delta X_{2}\left(t, t_{i}\right]\right)\right) d t\right)^{\beta / 2} \leq c \delta_{n}^{\gamma_{X} \beta / 2} .
\end{aligned}
$$

The last step follows from (A1). If $1<\beta<2$, we use the inequality $\left|x^{\beta}-y^{\beta}\right| \leq$ $\beta(x \vee y)^{\beta-1}|y-x|$ for positive $x, y$ and Hölder's inequality to obtain

$$
\begin{aligned}
& \mathbb{E}\left[\left|\left\|X_{1}^{(p)}-X_{2}^{(p)}\right\|_{2}^{\beta}-\left\|X_{1}-X_{2}\right\|_{2}^{\beta}\right|\right] \\
& \leq c \mathbb{E}\left[\left(\left\|X_{1}^{(p)}-X_{2}^{(p)}\right\|_{2}^{\beta-1} \vee\left\|X_{1}-X_{2}\right\|_{2}^{\beta-1}\right)\left|\left\|X_{1}^{(p)}-X_{2}^{(p)}\right\|_{2}-\left\|X_{1}-X_{2}\right\|_{2}\right|\right] \\
& \leq c \mathbb{E}\left[\left(\left\|X_{1}^{(p)}-X_{2}^{(p)}\right\|_{2}^{\beta-1} \vee\left\|X_{1}-X_{2}\right\|_{2}^{\beta-1}\right)\left\|\left(X_{1}^{(p)}-X_{2}^{(p)}\right)-\left(X_{1}-X_{2}\right)\right\|_{2}\right] \\
& \leq c\left(\mathbb{E}\left[\left\|X_{1}^{(p)}-X_{2}^{(p)}\right\|_{2}^{2} \vee\left\|X_{1}-X_{2}\right\|_{2}^{2}\right]\right)^{(\beta-1) / 2} \\
& \quad \times\left(\mathbb{E}\left[\left\|\left(X_{1}^{(p)}-X_{1}\right)-\left(X_{2}^{(p)}-X_{2}\right)\right\|_{2}^{2 /(3-\beta)}\right]\right)^{(3-\beta) / 2}=c P_{1} P_{2} \cdot(7.5)
\end{aligned}
$$

Since $(3-\beta)^{-1}<1$ the same arguments as in the case $0<\beta<1$ yield $P_{2} \leq c \delta_{n}^{\gamma x / 2}$. Moreover, we have

$$
P_{1}^{2 /(\beta-1)} \leq \mathbb{E}\left[\left\|X_{1}^{(p)}-X_{2}^{(p)}\right\|_{2}^{2}\right]+\mathbb{E}\left[\left\|X_{1}-X_{2}\right\|_{2}^{2}\right]=P_{11}+P_{12} .
$$

It follows from Remark 2.1 that $P_{12}<\infty$ and a similar argument yields $P_{11}<$ $\infty$.

Summarizing the previous bounds for $0<\beta<2$ under (A1), we have

$$
\mathbb{E}\left[I_{11}\right] \leq c \delta_{n}^{\left(\gamma_{X} \wedge \gamma_{Y}\right)(\beta \wedge 1) / 2}
$$

Now we turn to $I_{2}$. Observe that

$$
\begin{array}{r}
\left|I_{2}\right| \leq \frac{1}{n^{2}} \sum_{k, l=1}^{n}\left|\left\|X_{k}^{(p)}-X_{l}^{(p)}\right\|_{2}^{\beta}-\left\|X_{k}-X_{l}\right\|_{2}^{\beta}\right| \frac{1}{n^{2}} \sum_{k, l=1}^{n}\left\|Y_{k}^{(p)}-Y_{l}^{(p)}\right\|_{2}^{\beta} \\
+\frac{1}{n^{2}} \sum_{k, l=1}^{n}\left\|X_{k}-X_{l}\right\|_{2}^{\beta} \frac{1}{n^{2}} \sum_{k, l=1}^{n}\left|\left\|Y_{k}^{(p)}-Y_{l}^{(p)}\right\|_{2}^{\beta}-\left\|Y_{k}-Y_{l}\right\|_{2}^{\beta}\right|,
\end{array}
$$

and a similar bound exists for $\left|I_{3}\right|$. The same arguments as above yield

$$
\mathbb{E}\left[\left|I_{2}+I_{3}\right|\right] \leq c \delta_{n}^{\left(\gamma \times \wedge \gamma_{Y}\right)(\beta \wedge 1) / 2} .
$$

We omit further details.

Next assume that $(X, Y)$ have finite $\beta$ th moment for some $\beta \in(0,2)$. We follow the patterns of the proof in the finite variance case. We start by bounding 
$\mathbb{E}\left[\left|I_{1}\right|\right]$. First assume $\beta \in(0,1]$. Following (7.4), we have by (B2),

$$
\begin{aligned}
& \mathbb{E}\left[\left(\sum_{i=1}^{p} \int_{\Delta_{i}}\left(\Delta X_{1}\left(t, t_{i}\right]-\Delta X_{2}\left(t, t_{i}\right]\right)^{2} d t\right)^{\beta / 2}\right] \\
& \leq c \sum_{i=1}^{p}\left|\Delta_{i}\right|^{\beta / 2} \mathbb{E}\left[\max _{t \in \Delta_{i}}\left|\Delta X\left(t, t_{i}\right]\right|^{\beta}\right] \leq c p \delta_{n}^{\beta / 2+\gamma_{X}} .
\end{aligned}
$$

Now assume $1<\beta<2$. Following (7.5), we have by Hölder's inequality,

$$
\begin{aligned}
& \mathbb{E}\left[\left|\left\|X_{1}^{(p)}-X_{2}^{(p)}\right\|_{2}^{\beta}-\left\|X_{1}-X_{2}\right\|_{2}^{\beta}\right|\right] \\
& \leq c \mathbb{E}\left[\left(\left\|X_{1}^{(p)}-X_{2}^{(p)}\right\|_{2}^{\beta-1} \vee\left\|X_{1}-X_{2}\right\|_{2}^{\beta-1}\right)\left\|\left(X_{1}^{(p)}-X_{1}\right)-\left(X_{2}^{(p)}-X_{2}\right)\right\|_{2}\right] \\
& \leq c\left(\mathbb{E}\left[\left\|X_{1}^{(p)}-X_{2}^{(p)}\right\|_{2}^{\beta} \vee\left\|X_{1}-X_{2}\right\|_{2}^{\beta}\right]\right)^{(\beta-1) / \beta} \\
& \quad \times\left(\mathbb{E}\left[\left\|\left(X_{1}^{(p)}-X_{1}\right)-\left(X_{2}^{(p)}-X_{2}\right)\right\|_{2}^{\beta}\right]\right)^{1 / \beta} \\
& =c \widetilde{P}_{1} \widetilde{P}_{2} .
\end{aligned}
$$

Proceeding as for $0<\beta<1$, we have

$$
\widetilde{P}_{2}=\left(\mathbb{E}\left[\left\|\left(X_{1}^{(p)}-X_{1}\right)-\left(X_{2}^{(p)}-X_{2}\right)\right\|_{2}^{\beta}\right]\right)^{1 / \beta} \leq c\left(p \delta_{n}^{\beta / 2+\gamma_{X}}\right)^{1 / \beta} .
$$

We also have

$$
\widetilde{P}_{1}^{\beta /(\beta-1)} \leq \mathbb{E}\left[\left\|X_{1}^{(p)}-X_{2}^{(p)}\right\|_{2}^{\beta}\right]+\mathbb{E}\left[\left\|X_{1}-X_{2}\right\|_{2}^{\beta}\right] .
$$

The right-hand side is finite by assumption (B1). Collecting bounds for $0<\beta<$ 2 , we arrive at

$$
\mathbb{E}\left[\left|I_{1}\right|\right] \leq c\left(p \delta_{n}^{\beta / 2+\gamma_{X} \wedge \gamma_{Y}}\right)^{1 \wedge \beta^{-1}} .
$$

The quantities $\mathbb{E}\left[\left|I_{i}\right|\right], i=2,3$, can be bounded in a similar way.

Now we can finish the proof of the first two parts of Theorem 3.1. We assume that either (A1) or [(B1),(B2) and $\left.p \delta_{n}^{\beta / 2+\gamma_{X} \wedge \gamma_{Y}} \rightarrow 0\right]$ are satisfied. Under these assumptions, it follows from Proposition 7.1 that $T_{n, \beta}(X, Y)-$ $T_{n, \beta}\left(X^{(p)}, Y^{(p)}\right) \stackrel{\mathbb{P}}{\rightarrow} 0$. The quantity $T_{n, \beta}(X, Y)$ can be written as a $V$-statistic of order 4 of the sample $\left(\left(X_{i}, Y_{i}\right)\right)_{i=1, \ldots, n}$; see Appendix A. (Lyons (2013) used a $V$-statistics of order 6 . The higher order leads to a higher numerical complexity for the calculation of the bootstrap quantities.) Since $X, Y$ are assumed independent and $\mathbb{E}\left[\|X\|_{2}^{\beta}\right]+\mathbb{E}\left[\|Y\|_{2}^{\beta}\right]<\infty$ (see Remark 2.1) we may apply the strong law of large numbers to the $V$-statistic $T_{n, \beta}(X, Y)$ implying that

$$
T_{n, \beta}(X, Y) \stackrel{\text { a.s. }}{\rightarrow} T_{\beta}(X, Y)=0 .
$$

Hence the first parts of the theorem follow.

imsart-generic ver. 2014/10/16 file: dmmsl2018main_2ndsubmissionArxiv.tex date: November 30, 2018 
Under the corresponding growth conditions (A2) and (B4) on $\delta_{n} \rightarrow 0$, Proposition 7.1 also yields $n\left(T_{n, \beta}(X, Y)-T_{n, \beta}\left(X^{(p)}, Y^{(p)}\right)\right) \stackrel{\mathbb{P}}{\rightarrow} 0$. Then we can use the fact that the $V$-statistic $T_{n, \beta}(X, Y)$ is degenerate of order 1 to conclude that $n T_{n, \beta}(X, Y)$ converges in distribution to a series of independent weighted $\chi^{2}$ distributed random variables, and $n T_{n, \beta}\left(X^{(p)}, Y^{(p)}\right)$ has the same weak limit; we refer to Arcones and Giné (1992), Serfling (1980) for general limit theory on $U$ - and $V$-statistics.

Next we prove (3) and (4). In view of the first two parts (1), (2) of the theorem they will follow if we can show consistency of $T_{n, \beta}\left(X^{(p)}, X^{(p)}\right)$ and $T_{n, \beta}\left(Y^{(p)}, Y^{(p)}\right)$. This is the content of the following lemma.

Lemma 7.2. Assume the following conditions:

1. $X$ is defined on $[0,1]$ and has Riemann square-integrable sample paths.

2. If $X$ has a finite first moment $X$ is centered.

3. $\delta_{n} \rightarrow 0$ as $n \rightarrow \infty$.

4. $\beta \in(0,2)$.

Moreover, consider the following conditions:

(1) $X$ has finite second moment and there exist $\gamma_{X}>0$ and $c>0$ such that

$$
\operatorname{var}(X(s, t]) \leq c|t-s|^{\gamma_{X}}, \quad s<t .
$$

If $\beta \in(1,2)$ we also assume

$$
\max _{0 \leq t \leq 1} \mathbb{E}\left[|X(t)|^{2(2 \beta-1)}\right]<\infty .
$$

(2) For some $\beta \in(0,1)$,

$$
\mathbb{E}\left[\max _{0 \leq t \leq 1}|X(t)|^{2 \beta}\right]<\infty,
$$

and there exist $\gamma_{X}^{\prime}>0$ and $c>0$ such that

$$
\max _{i=1, \ldots, p} \mathbb{E}\left[\max _{t \in \Delta_{i}}\left|\Delta X\left(t, t_{i}\right]\right|^{2 \beta}\right] \leq c \delta_{n}^{\gamma_{X}^{\prime}}
$$

and $p \delta_{n}^{\beta+\gamma_{X}^{\prime}} \rightarrow 0$.

If either (1) or (2) hold then

$$
T_{n, \beta}\left(X^{(p)}, X^{(p)}\right)-T_{n, \beta}(X, X) \stackrel{\mathbb{P}}{\rightarrow} 0 .
$$

Moreover, we also have

$$
T_{n, \beta}\left(X^{(p)}, X^{(p)}\right) \stackrel{\mathbb{P}}{\rightarrow} T_{\beta}(X, X),
$$

where

$T_{\beta}(X, X)=\mathbb{E}\left[\left\|X_{1}-X_{2}\right\|_{2}^{2 \beta}\right]+\left(\mathbb{E}\left[\left\|X_{1}-X_{2}\right\|_{2}^{\beta}\right]\right)^{2}-2 \mathbb{E}\left[\left\|X_{1}-X_{2}\right\|_{2}^{\beta}\left\|X_{1}-X_{3}\right\|_{2}^{\beta}\right]$. 
Note that (7.7) and (7.8) are contained in conditions (A1) and (A3), respectively, while (7.9) and (7.10) are contained in (B3). Therefore the conditions of Lemma 7.2 are satisfied if those of Theorem 3.1, (3) and (4), hold.

Proof. We assume condition (1). We use the decomposition (7.1) and follow the lines of the proof of Proposition 7.1 In this case,

$$
\begin{aligned}
I_{1}= & \frac{1}{n^{2}} \sum_{k, l=1}^{n}\left(\left\|X_{k}^{(p)}-X_{l}^{(p)}\right\|_{2}^{2 \beta}-\left\|X_{k}-X_{l}\right\|_{2}^{2 \beta}\right) \\
I_{2}= & \left(\frac{1}{n^{2}} \sum_{k, l=1}^{n}\left\|X_{k}^{(p)}-X_{l}^{(p)}\right\|_{2}^{\beta}\right)^{2}-\left(\frac{1}{n^{2}} \sum_{k, l=1}^{n}\left\|X_{k}-X_{l}\right\|_{2}^{\beta}\right)^{2} \\
I_{3}= & \frac{1}{n^{3}} \sum_{k, l, m=1}^{n}\left(\left\|X_{k}^{(p)}-X_{l}^{(p)}\right\|_{2}^{\beta}\left\|X_{k}^{(p)}-X_{m}^{(p)}\right\|_{2}^{\beta}\right. \\
& \left.\quad-\left\|X_{k}-X_{l}\right\|_{2}^{\beta}\left\|X_{k}-X_{m}\right\|_{2}^{\beta}\right) .
\end{aligned}
$$

We start by considering $I_{1}$. First assume that $\beta \leq 1$. Observe that

$$
\begin{aligned}
\mathbb{E}\left[\left|I_{1}\right|\right] \leq & \mathbb{E}\left[\left\|\left(X_{1}^{(p)}-X_{1}\right)-\left(X_{2}^{(p)}-X_{2}\right)\right\|_{2}^{\beta}\left(\left\|X_{1}^{(p)}-X_{2}^{(p)}\right\|_{2}^{\beta}+\left\|X_{1}-X_{2}\right\|_{2}^{\beta}\right)\right] \\
\leq & \left(\mathbb{E}\left[\left\|\left(X_{1}^{(p)}-X_{1}\right)-\left(X_{2}^{(p)}-X_{2}\right)\right\|_{2}^{2 \beta}\right]\right)^{1 / 2} \\
& \times\left(\left(\mathbb{E}\left[\left\|X_{1}^{(p)}-X_{2}^{(p)}\right\|_{2}^{2 \beta}\right)^{1 / 2}+\left(\mathbb{E}\left\|X_{1}-X_{2}\right\|_{2}^{2 \beta}\right]\right)^{1 / 2}\right) .
\end{aligned}
$$

Similarly as in (7.4) the first expectation is bounded by $c \delta_{n}^{\beta \gamma}$, while the remaining two expectations are bounded, so that as in the proof of Proposition 7.1 , we have that

$$
\mathbb{E}\left[\left|I_{1}\right|\right] \leq c \delta_{n}^{\beta \gamma_{X} / 2} .
$$

If $1<\beta<2$ we may proceed as for $\mathbb{E}\left[I_{11}\right]$ in the proof of Proposition 7.1 in the case $1<\beta<2$ :

$$
\begin{aligned}
\mathbb{E}\left[\left|I_{1}\right|\right] \leq & \mathbb{E}\left[\left|\left\|X_{1}^{(p)}-X_{2}^{(p)}\right\|_{2}^{2 \beta}-\left\|X_{1}-X_{2}\right\|_{2}^{2 \beta}\right|\right] \\
\leq & c \mathbb{E}\left[\left\|X_{1}^{(p)}-X_{2}^{(p)}\right\|_{2}^{2 \beta-1} \vee\left\|X_{1}-X_{2}\right\|_{2}^{2 \beta-1}\right. \\
& \left.\quad \times\left|\left\|X_{1}^{(p)}-X_{2}^{(p)}\right\|_{2}-\left\|X_{1}-X_{2}\right\|_{2}\right|\right] \\
\leq & c\left(\mathbb{E}\left[\left\|X_{1}^{(p)}-X_{2}^{(p)}\right\|_{2}^{2(2 \beta-1)} \vee\left\|X_{1}-X_{2}\right\|_{2}^{2(2 \beta-1)}\right]\right)^{1 / 2} \\
& \times\left(\mathbb{E}\left[\left\|\left(X_{1}^{(p)}-X_{1}\right)-\left(X_{2}^{(p)}-X_{2}\right)\right\|_{2}^{2}\right]\right)^{1 / 2} \\
= & c P_{1} P_{2} .
\end{aligned}
$$

We have $P_{2} \leq c \delta_{n}^{\gamma_{X} / 2}$ and

$$
P_{1}^{2} \leq \mathbb{E}\left[\left\|X_{1}^{(p)}-X_{2}^{(p)}\right\|_{2}^{2(2 \beta-1)}\right]+\mathbb{E}\left[\left\|X_{1}-X_{2}\right\|_{2}^{2(2 \beta-1)}\right]=P_{11}+P_{12} .
$$


We deal only with $P_{12} ; P_{11}$ can be bounded in a similar way. For $1<2 \beta \leq 2$, the function $f(x)=|x|^{2 \beta-1}$ is concave. Therefore

$$
\begin{aligned}
P_{12} & =\mathbb{E}\left[\left(\int_{0}^{1}\left(X_{1}(t)-X_{2}(t)\right)^{2} d t\right)^{2 \beta-1}\right] \\
& \leq\left(\mathbb{E}\left[\int_{0}^{1}\left(X_{1}(t)-X_{2}(t)\right)^{2} d t\right]\right)^{2 \beta-1}<\infty .
\end{aligned}
$$

In the last step we used (7.7).

If $2<2 \beta<4$ we have by Lyapunov's inequality and (7.8),

$$
\begin{aligned}
P_{12} & =\mathbb{E}\left[\left(\int_{0}^{1}\left(X_{1}(t)-X_{2}(t)\right)^{2} d t\right)^{2 \beta-1}\right] \\
& \leq \mathbb{E}\left[\int_{0}^{1}\left|X_{1}(t)-X_{2}(t)\right|^{2(2 \beta-1)} d t\right]<\infty .
\end{aligned}
$$

Thus we proved that

$$
\mathbb{E}\left[\left|I_{1}\right|\right] \leq c \delta_{n}^{\gamma_{X}(\beta \wedge 1) / 2} .
$$

We can deal with $I_{2}$ in the same way by observing that

$$
\begin{aligned}
I_{2}= & \frac{1}{n^{2}} \sum_{k, l=1}^{n}\left(\left\|X_{k}^{(p)}-X_{l}^{(p)}\right\|_{2}^{\beta}-\left\|X_{k}-X_{l}\right\|_{2}^{\beta}\right) \\
& \quad \times \frac{1}{n^{2}} \sum_{k, l=1}^{n}\left(\left\|X_{k}^{(p)}-X_{l}^{(p)}\right\|_{2}^{\beta}+\left\|X_{k}-X_{l}\right\|_{2}^{\beta}\right) \\
= & \widetilde{P}_{1} \widetilde{P}_{2} .
\end{aligned}
$$

The expected value of $\widetilde{P}_{2}$ is bounded and hence $\widetilde{P}_{2}$ is stochastically bounded while similar calculations as for $I_{1}$ show that $\mathbb{E}\left[\left|\widetilde{P}_{1}\right|\right] \rightarrow 0$. Hence $I_{2} \stackrel{\mathbb{P}}{\rightarrow} 0$. We have

$$
\begin{aligned}
I_{3}= & \frac{1}{n^{3}} \sum_{k, l, m=1}^{n}\left(\left\|X_{k}^{(p)}-X_{l}^{(p)}\right\|_{2}^{\beta}-\left\|X_{k}-X_{l}\right\|_{2}^{\beta}\right)\left\|X_{k}^{(p)}-X_{m}^{(p)}\right\|_{2}^{\beta} \\
& +\frac{1}{n^{3}} \sum_{k, l, m=1}^{n}\left\|X_{k}-X_{l}\right\|_{2}^{\beta}\left(\left\|X_{k}^{(p)}-X_{m}^{(p)}\right\|_{2}^{\beta}-\left\|X_{k}-X_{m}\right\|_{2}^{\beta}\right) \\
= & I_{31}+I_{32} .
\end{aligned}
$$

We will deal only with $I_{32}$; the other case is similar. Assume $0<\beta \leq 1$. By the Cauchy-Schwarz inequality and using similar bounds as above,

$$
\begin{aligned}
\mathbb{E}\left[\left|I_{32}\right|\right] & \leq\left(\mathbb{E}\left[\left\|X_{1}-X_{2}\right\|_{2}^{2 \beta}\right]\right)^{1 / 2}\left(\mathbb{E}\left[\left|\left\|X_{1}^{(p)}-X_{3}^{(p)}\right\|_{2}^{\beta}-\left\|X_{1}-X_{3}\right\|_{2}^{\beta}\right|^{2}\right]\right)^{1 / 2} \\
& \leq\left(\mathbb{E}\left[\left\|X_{1}-X_{2}\right\|_{2}^{2 \beta}\right]\right)^{1 / 2}\left(\mathbb{E}\left[\left\|\left(X_{1}^{(p)}-X_{1}\right)-\left(X_{3}^{(p)}-X_{3}\right)\right\|_{2}^{2 \beta}\right]\right)^{1 / 2}
\end{aligned}
$$

imsart-generic ver. 2014/10/16 file: dmms12018main_2ndsubmissionArxiv.tex date: November 30, 2018 
$\rightarrow 0$.

Now assume $1<\beta<2$. Then

$$
\begin{aligned}
\mathbb{E}\left[\left|I_{32}\right|\right] \leq & c \mathbb{E}\left[\left\|\left(X_{1}^{(p)}-X_{1}\right)-\left(X_{3}^{(p)}-X_{3}\right)\right\|_{2}\right. \\
& \left.\times\left(\left\|X_{1}^{(p)}-X_{3}^{(p)}\right\|_{2}^{\beta-1} \vee\left\|X_{1}-X_{3}\right\|_{2}^{\beta-1}\right)\left\|X_{1}-X_{2}\right\|_{2}^{\beta}\right] \\
\leq & c\left(\mathbb{E}\left[\left\|\left(X_{1}^{(p)}-X_{1}\right)-\left(X_{3}^{(p)}-X_{3}\right)\right\|_{2}^{2}\right]\right)^{1 / 2} \\
& \times\left(\mathbb{E}\left[\left(\left\|X_{1}^{(p)}-X_{3}^{(p)}\right\|_{2}^{2(\beta-1)} \vee\left\|X_{1}-X_{3}\right\|_{2}^{2(\beta-1)}\right)\left\|X_{1}-X_{2}\right\|_{2}^{2 \beta}\right]\right)^{1 / 2} \\
\leq & c\left(\mathbb{E}\left[\left\|\left(X_{1}^{(p)}-X_{1}\right)-\left(X_{3}^{(p)}-X_{3}\right)\right\|_{2}^{2}\right]\right)^{1 / 2} \\
& \times\left\{\left(\mathbb{E}\left[\left\|X_{1}^{(p)}-X_{3}^{(p)}\right\|_{2}^{2(\beta-1)}\left\|X_{1}-X_{2}\right\|_{2}^{2 \beta}\right]\right)^{1 / 2}\right. \\
& \left.+\left(\mathbb{E}\left[\left\|X_{1}-X_{3}\right\|_{2}^{2(\beta-1)}\left\|X_{1}-X_{2}\right\|_{2}^{2 \beta}\right]\right)^{1 / 2}\right\} .
\end{aligned}
$$

The first factor is $P_{2}$ from above which is bounded by $c \delta_{n}^{\gamma_{X} / 2}$. For the second term, we only consider $\mathbb{E}\left[\left\|X_{1}^{(p)}-X_{3}^{(p)}\right\|_{2}^{2(\beta-1)}\left\|X_{1}-X_{2}\right\|_{2}^{2 \beta}\right]$ by a symmetry argument. An application of Hölder's inequality to this quantity yields the bounds

$$
\left(\mathbb{E}\left[\left\|X_{1}^{(p)}-X_{3}^{(p)}\right\|_{2}^{2(2 \beta-1)}\right]\right)^{\frac{\beta-1}{2 \beta-1}}\left(\mathbb{E}\left[\left\|X_{1}-X_{2}\right\|_{2}^{2(2 \beta-1)}\right]\right)^{\frac{\beta}{2 \beta-1}}=P_{11}^{\frac{\beta-1}{2 \beta-1}} P_{12}^{\frac{\beta}{2 \beta-1}},
$$

where $P_{11}, P_{12}$ are defined above and shown to be bounded. This concludes the proof under condition (1).

We assume condition (2). Now we prove the lemma under the condition that the moments of $X(t)$ of the order $2 \beta \in(0,2)$ are finite. We have for $2 \beta \leq 1$ by concavity and in view of condition (7.10),

$$
\begin{aligned}
\mathbb{E}\left[\left|I_{1}\right|\right] & \leq \mathbb{E}\left[\left\|\left(X_{1}-X_{1}^{(p)}\right)-\left(X_{2}-X_{2}^{(p)}\right)\right\|_{2}^{2 \beta}\right] \\
& \leq c \delta_{n}^{\beta} \sum_{i=1}^{p} \mathbb{E}\left[\max _{t \in \Delta_{i}}\left|\Delta X\left(t, t_{i}\right]\right|^{2 \beta}\right] \leq c p \delta_{n}^{\beta+\gamma_{X}^{\prime}} .
\end{aligned}
$$

The right-hand side goes to zero by assumption. For $2 \beta \in(1,2)$ we have by Hölder's inequality,

$$
\begin{aligned}
\mathbb{E}\left[\left|I_{1}\right|\right] \leq & \mathbb{E}\left[\left|\left\|X_{1}^{(p)}-X_{2}^{(p)}\right\|_{2}^{2 \beta}-\left\|X_{1}-X_{2}\right\|_{2}^{2 \beta}\right|\right] \\
\leq & c \mathbb{E}\left[\left(\left\|X_{1}^{(p)}-X_{2}^{(p)}\right\|_{2}^{2 \beta-1} \vee\left\|X_{1}-X_{2}\right\|_{2}^{2 \beta-1}\right)\right. \\
& \left.\quad \times\left\|\left(X_{1}^{(p)}-X_{1}\right)-\left(X_{2}^{(p)}-X_{2}\right)\right\|_{2}\right] \\
\leq & c\left(\mathbb{E}\left[\left\|X_{1}^{(p)}-X_{2}^{(p)}\right\|_{2}^{2 \beta} \vee\left\|X_{1}-X_{2}\right\|_{2}^{2 \beta}\right]\right)^{(2 \beta-1) /(2 \beta)} \\
& \times\left(\mathbb{E}\left[\left\|\left(X_{1}^{(p)}-X_{1}\right)-\left(X_{2}^{(p)}-X_{2}\right)\right\|_{2}^{2 \beta}\right]\right)^{1 /(2 \beta)}
\end{aligned}
$$

imsart-generic ver. 2014/10/16 file: dmms12018main_2ndsubmissionArxiv.tex date: November 30, 2018 


$$
=c \widehat{P}_{1} \widehat{P}_{2} \text {. }
$$

The quantity $\widehat{P}_{1}$ is finite in view of $(7.9)$ and $\widehat{P}_{2} \rightarrow 0$ by the argument of (7.18).

For $I_{2}=\widetilde{P}_{1} \widetilde{P}_{2}$ we use $(7.15)$. Since $\mathbb{E}\left[\left\|X_{1}-X_{2}\right\|_{2}^{\beta}\right]$ and $\mathbb{E}\left[\left\|X_{1}^{(p)}-X_{2}^{(p)}\right\|_{2}^{\beta}\right]$ are finite the expectation of $\widetilde{P}_{2}$ is bounded while

$$
\mathbb{E}\left[\left|\widetilde{P}_{1}\right|\right] \leq 2 \mathbb{E}\left[\left\|X-X^{(p)}\right\|_{2}^{\beta}\right] \leq 2\left(\mathbb{E}\left[\left\|X-X^{(p)}\right\|_{2}^{2 \beta}\right]\right)^{1 / 2} .
$$

The argument of (7.18) shows that the right-hand side converges to zero.

Finally, we use the decomposition $I_{3}=I_{31}+I_{32}$. Inequality (7.17) and the bounds above show that $\mathbb{E}\left[\left|I_{32}\right|\right] \rightarrow 0$; the case $\mathbb{E}\left[\left|I_{31}\right|\right] \rightarrow 0$ follows in a similar way.

Collecting all bounds above, we proved $T_{n, \beta}\left(X^{(p)}, X^{(p)}\right)-T_{n, \beta}(X, X) \stackrel{\mathbb{P}}{\rightarrow} 0$ both under the conditions of (1) and (2). Then relation (7.11) is immediate. Indeed, under the assumption $\mathbb{E}\left[\|X\|_{2}^{2 \beta}\right]<\infty$ the strong law of large numbers for $U$ - and $V$-statistics yields $T_{n, \beta}(X, X) \stackrel{\text { a.s. }}{\rightarrow} T_{\beta}(X, X)$.

\section{Appendix A: The sample distance covariance as a degenerate V-statistic}

We assume that $Z_{i}=\left(X_{i}, Y_{i}\right), i=1,2, \ldots$, is an iid sequence with generic element $(X, Y)$ whose components are Riemann square-integrable on $[0,1]$, and $\mathbb{E}\left[\|X\|_{2}^{\beta}+\|Y\|_{2}^{\beta}+\|X\|_{2}^{\beta}\|Y\|_{2}^{\beta}\right]<\infty$ and for some $\beta \in(0,2)$. Under the assumption of independence on $X, Y$ Lyons $(2013,2018)$ proved that $T_{n, \beta}(X, Y)$ has representation as a $V$-statistic of order 6 with degenerate kernel of order 1 . In what follows, we will indicate that it can be written as a $V$-statistic of order 4 with symmetric degenerate kernel of order 1 . This fact is useful for improving upon the complexity of the numerical approximation of the sample distance correlation and its bootstrap version.

We start with the kernel

$$
\begin{aligned}
& f\left(\left(x_{1}, y_{1}\right),\left(x_{2}, y_{2}\right),\left(x_{3}, y_{3}\right),\left(x_{4}, y_{4}\right)\right)\left(=: f\left(z_{1}, z_{2}, z_{3} . z_{4}\right)\right) \\
& \quad=\left\|x_{1}-x_{2}\right\|_{2}^{\beta}\left\|y_{1}-y_{2}\right\|_{2}^{\beta}+\left\|x_{1}-x_{2}\right\|_{2}^{\beta}\left\|y_{3}-y_{4}\right\|_{2}^{\beta}-2\left\|x_{1}-x_{2}\right\|_{2}^{\beta}\left\|y_{1}-y_{3}\right\|_{2}^{\beta} .
\end{aligned}
$$

From this representation, it is obvious that

$$
T_{n, \beta}(X, Y)=\frac{1}{n^{4}} \sum_{1 \leq i, j, k, l \leq n} f\left(Z_{i}, Z_{j}, Z_{k}, Z_{l}\right) .
$$

Then one can define the corresponding symmetric kernel via the usual symmetrization as

$$
h\left(z_{1}, z_{2}, z_{3}, z_{4}\right)=\frac{1}{24} \sum_{\left(l_{1}, l_{2}, l_{3}, l_{4}\right)} \sum_{(1,2,3,4)} f\left(z_{l_{1}}, z_{l_{2}}, z_{l_{3}}, z_{l_{4}}\right) .
$$


It is not difficult to see that the kernel $h$ is at least 1-degenerate, by showing that, under the null hypothesis of independence of $X$ and $Y$,

$$
\begin{aligned}
& \mathbb{E}\left[f\left(z_{1}, Z_{2}, Z_{3}, Z_{4}\right)\right]+\mathbb{E}\left[f\left(Z_{2}, z_{1}, Z_{3}, Z_{4}\right)\right]+\mathbb{E}\left[f\left(Z_{2}, Z_{3}, z_{1}, Z_{4}\right)\right] \\
& \quad+\mathbb{E}\left[f\left(Z_{2}, Z_{3}, Z_{4}, z_{1}\right)\right]=0 .
\end{aligned}
$$

Still under the null hypothesis of independence of $X$ and $Y$,

$$
\begin{aligned}
\mathbb{E}[ & \left.h\left(z_{1}, z_{2},\left(X_{3}, Y_{3}\right),\left(X_{4}, Y_{4}\right)\right)\right] \\
= & \frac{1}{6}\left(\left\|x_{1}-x_{2}\right\|_{2}^{\beta}+\mathbb{E}\left[\left\|X_{1}-X_{2}\right\|_{2}^{\beta}\right]-\mathbb{E}\left[\left\|x_{1}-X\right\|_{2}^{\beta}\right]-\mathbb{E}\left[\left\|x_{2}-X\right\|_{2}^{\beta}\right]\right) \\
\quad & \quad\left(\left\|y_{1}-y_{2}\right\|_{2}^{\beta}+\mathbb{E}\left[\left\|Y_{1}-Y_{2}\right\|_{2}^{\beta}\right]-\mathbb{E}\left[\left\|y_{1}-Y\right\|_{2}^{\beta}\right]-\mathbb{E}\left[\left\|y_{2}-Y\right\|_{2}^{\beta}\right]\right),
\end{aligned}
$$

and the right-hand side is not constant. Hence, the kernel $h$ is precisely 1degenerate. In summary:

Lemma A.1. If $X, Y$ are independent and $\mathbb{E}\left[\|X\|_{2}^{\beta}+\|Y\|_{2}^{\beta}\right]<\infty$ for some $\beta \in(0,2)$ then $T_{n, \beta}(X, Y)$ has representation as a $V$-statistic with a symmetric kernel $h$ of order 4 which is 1-degenerate. Moreover, the corresponding $U$ statistic $\widetilde{T}_{n, \beta}(X, Y)$, which is obtained from $T_{n, \beta}(X, Y)$ by restricting the summation to indices $\left(i_{1}, i_{2}, i_{3}, i_{4}\right)$ with mutually distinct components, satisfies the relation that as $n \rightarrow \infty$

$$
n\left(T_{n, \beta}(X, Y)-\widetilde{T}_{n, \beta}(X, Y)\right) \stackrel{\mathbb{P}}{\rightarrow} \mathbb{E}\left[\left\|X_{1}-X_{2}\right\|_{2}^{\beta}\right] \mathbb{E}\left[\left\|Y_{1}-Y_{2}\right\|_{2}^{\beta}\right] .
$$

Indeed, observe that $\Delta_{n}=T_{n, \beta}-\widetilde{T}_{n, \beta}$ is based on summation of the kernel $h$ over indices $\left(i_{1}, i_{2}, i_{3}, i_{4}\right)$ for which at least two components coincide. If more than 2 indices coincide the number of these summands in $\Delta_{n}$ is of the order $O\left(n^{2}\right)$. However, the normalization in $n \Delta_{n}$ is of the order $n^{3}$. Therefore the sum of these terms is negligible as $n \rightarrow \infty$. Finally, the part of the sum corresponding to the case when exactly two indices coincide and the other indices are different, can be written as a $U$-statistic of order 3 . By the law of large numbers, this $U$ statistic converges a.s. to $\mathbb{E}\left[\left\|X_{1}-X_{2}\right\|_{2}^{\beta}\right] \mathbb{E}\left[\left\|Y_{1}-Y_{2}\right\|_{2}^{\beta}\right]$.

Remark A.2. The additional moment assumption on $h\left(Z_{i_{1}}, Z_{i_{2}}, Z_{i_{3}}, Z_{i_{4}}\right), 1 \leq$ $i_{1} \leq i_{2} \leq i_{3} \leq i_{4} \leq 4$, required in Corollary 5.2 is satisfied for our kernel. Note that it suffices to consider the non-symmetric kernel $f$, and to show that $\mathbb{E}\left[\left(f\left(Z_{i_{1}}, Z_{i_{2}}, Z_{i_{3}}, Z_{i_{4}}\right)\right)^{2}\right]<\infty$, for all indices $1 \leq i_{1}, \ldots, i_{4} \leq 4$. For our specific kernel, this condition reads

$$
\mathbb{E}\left[\left(\left\|X_{i_{1}}-X_{i_{2}}\right\|^{\beta}\left[\left\|Y_{i_{1}}-Y_{i_{2}}\right\|^{\beta}+\left\|Y_{i_{3}}-Y_{i_{4}}\right\|^{\beta}-2\left\|Y_{i_{1}}-Y_{i_{3}}\right\|^{\beta}\right]\right)^{2}\right]<\infty,
$$

and this holds under the moment conditions made in this paper.

\section{Appendix B: Bootstrap consistency for Section 5}

For the proof of the bootstrap consistency in Section 5 we need a.s. convergence of $T_{n, 1}\left(X^{(p)}, Y^{(p)}\right)=: T_{n}\left(X^{(p)}, Y^{(p)}\right)$. We give some sufficient conditions. 
Lemma B.1. Assume the following conditions on the Riemann square-integrable process $X$ on $[0,1]$.

1. $\mathbb{E}\left[\|X\|_{2}^{2}\right]<\infty$ and $\mathbb{E}[X(u)]=0$ for $u \in[0,1]$.

2. (A.1) holds.

3. $E\left[|X(t)-X(s)|^{4}\right] \leq c|t-s|^{\widetilde{\gamma}_{X}}$ holds for some $\widetilde{\gamma}_{X}>0$.

4. $\sum_{n=1}^{\infty} n^{-1}\left(\delta_{n}^{\gamma X}+\delta_{n}^{\widetilde{\gamma} X}\right)<\infty$.

Then $T_{n}\left(X^{(p)}, X^{(p)}\right) \stackrel{\text { a.s. }}{\rightarrow} T(X, X)$ holds as $n \rightarrow \infty$.

Proof. From (7.1) recall the decomposition $T_{n}\left(X^{(p)}, X^{(p)}\right)-T_{n}(X, X)=I_{1}+$ $I_{2}-2 I_{3}$; see also (7.12). Since $\mathbb{E}\left[\|X\|_{2}^{2}\right]<\infty$, by the strong law of large numbers for $V$-statistics, $T_{n}(X, X) \stackrel{\text { a.s. }}{\rightarrow} T(X, X)$. Therefore it suffices to show that

$$
\begin{aligned}
I_{i} & \stackrel{\text { a.s. }}{\rightarrow} 0, \quad i=1,3, \\
I_{2}^{\prime} & :=\frac{1}{n^{2}} \sum_{k, l=1}^{n}\left\|X_{k}^{(p)}-X_{l}^{(p)}\right\|_{2}-\frac{1}{n^{2}} \sum_{k, l=1}^{n}\left\|X_{k}-X_{l}\right\|_{2} \stackrel{\text { a.s. }}{\rightarrow} 0 .
\end{aligned}
$$

We have

$$
\begin{aligned}
\left|I_{2}^{\prime}\right| & \leq \frac{1}{n} \sum_{k}^{n}\left\|X_{k}^{(p)}-X_{k}\right\|_{2} \\
& =\frac{1}{n} \sum_{k}^{n}\left(\left\|X_{k}^{(p)}-X_{k}\right\|_{2}-\mathbb{E}\left[\left\|X^{(p)}-X\right\|_{2}\right]\right)+\mathbb{E}\left[\left\|X^{(p)}-X\right\|_{2}\right] .
\end{aligned}
$$

By Jensen's inequality,

$$
\mathbb{E}\left[\left\|X^{(p)}-X\right\|_{2}\right] \leq\left(\int_{0}^{1} \operatorname{var}\left(X^{(p)}(u)-X(u)\right) d u\right)^{1 / 2} \leq \delta_{n}^{\gamma X / 2} \rightarrow 0 .
$$

Moreover,

$$
\operatorname{var}\left(\frac{1}{n} \sum_{k}^{n}\left(\left\|X_{k}^{(p)}-X_{k}\right\|_{2}\right)\right) \leq n^{-1} \mathbb{E}\left[\left\|X^{(p)}-X\right\|_{2}^{2}\right] \leq n^{-1} \delta_{n}^{\gamma X} .
$$

Using Markov's inequality and the Borel-Cantelli lemma, we conclude that $I_{2}^{\prime} \stackrel{\text { a.s. }}{\rightarrow}$ 0 if $\sum_{n} n^{-1} \delta_{n}^{\gamma_{X}}<\infty$.

The proof of $I_{1} \stackrel{\text { a.s. }}{\rightarrow} 0$ is similar. We have by the Cauchy-Schwarz inequality,

$$
\begin{aligned}
\left|I_{1}\right| \leq & \left(\frac{1}{n^{2}} \sum_{k, l=1}^{n}\left(\left\|X_{k}^{(p)}-X_{l}^{(p)}\right\|_{2}-\left\|X_{k}-X_{l}\right\|_{2}\right)^{2}\right)^{1 / 2} \\
& \times\left(\frac{1}{n^{2}} \sum_{k, l=1}^{n}\left(\left\|X_{k}^{(p)}-X_{l}^{(p)}\right\|_{2}+\left\|X_{k}-X_{l}\right\|_{2}\right)^{2}\right)^{1 / 2} \\
\leq & c \frac{1}{n^{2}} \sum_{k, l=1}^{n}\left(\left\|X_{k}^{(p)}-X_{k}\right\|_{2}+\left\|X_{l}-X_{l}^{(p)}\right\|_{2}\right)^{2}
\end{aligned}
$$

imsart-generic ver. 2014/10/16 file: dmms12018main_2ndsubmissionArxiv.tex date: November 30, 2018 


$$
\begin{gathered}
+c\left(\frac{1}{n^{2}} \sum_{k, l=1}^{n}\left(\left\|X_{k}^{(p)}-X_{k}\right\|_{2}+\left\|X_{l}-X_{l}^{(p)}\right\|_{2}\right)^{2}\right)^{1 / 2} \\
\times\left(\frac{1}{n^{2}} \sum_{k, l=1}^{n}\left\|X_{k}-X_{l}\right\|_{2}^{2}\right)^{1 / 2} .
\end{gathered}
$$

Therefore it remains to show that

$$
\frac{1}{n} \sum_{k=1}^{n}\left(\left\|X_{k}^{(p)}-X_{k}\right\|_{2}^{2}-\mathbb{E}\left[\left\|X^{(p)}-X\right\|_{2}^{2}\right]\right)+\mathbb{E}\left[\left\|X^{(p)}-X\right\|_{2}^{2}\right] \stackrel{\text { a.s. }}{\rightarrow} 0 .
$$

But we have $\mathbb{E}\left[\left\|X^{(p)}-X\right\|_{2}^{2}\right]=O\left(\delta_{n}^{\gamma x}\right)$ and

$$
\begin{aligned}
\operatorname{var}\left(\frac{1}{n} \sum_{k=1}^{n}\left(\left\|X_{k}^{(p)}-X_{k}\right\|_{2}^{2}\right)\right) & \leq n^{-1} \mathbb{E}\left[\left\|X_{k}^{(p)}-X_{k}\right\|_{2}^{4}\right] \\
& \leq n^{-1} \int_{0}^{1} \mathbb{E}\left[\left(X^{(p)}(u)-X(u)\right)^{4}\right] d u \leq n^{-1} \delta_{n}^{\tilde{\gamma}_{X}} .
\end{aligned}
$$

Since we assume $\sum_{n} n^{-1} \delta_{n}^{\widetilde{\gamma} x}<\infty$ applications of Markov's inequality and the Borel-Cantelli lemma show that $I_{1} \stackrel{\text { a.s. }}{\rightarrow} 0$.

Finally, we show $I_{3} \stackrel{\text { a.s. }}{\rightarrow} 0$. We have

$$
\begin{aligned}
I_{3}= & \frac{1}{n^{3}} \sum_{k, l, m=1}^{n}\left(\left\|X_{k}^{(p)}-X_{l}^{(p)}\right\|_{2}-\left\|X_{k}-X_{l}\right\|_{2}\right) \\
& \times\left(\left\|X_{k}^{(p)}-X_{m}^{(p)}\right\|_{2}-\left\|X_{k}-X_{m}\right\|_{2}\right) \\
& +\frac{2}{n^{3}} \sum_{k, l, m=1}^{n}\left(\left\|X_{k}^{(p)}-X_{l}^{(p)}\right\|_{2}-\left\|X_{k}-X_{l}\right\|_{2}\right)\left\|X_{k}-X_{m}\right\|_{2} \\
= & I_{31}+I_{32} .
\end{aligned}
$$

The Cauchy-Schwarz inequality yields

$$
\begin{aligned}
\left|I_{31}\right| & \leq \frac{1}{n^{2}} \sum_{k, l=1}^{n}\left(\left\|X_{k}^{(p)}-X_{l}^{(p)}\right\|_{2}-\left\|X_{k}-X_{l}\right\|_{2}\right)^{2} \\
& \leq c \frac{1}{n} \sum_{k=1}^{n}\left\|X_{k}^{(p)}-X_{k}\right\|_{2}^{2} \stackrel{\text { a.s. }}{\rightarrow} 0, \\
\left|I_{32}\right| & \leq c\left(\frac{1}{n^{2}} \sum_{k, l=1}^{n}\left(\left\|X_{k}^{(p)}-X_{l}^{(p)}\right\|_{2}-\left\|X_{k}-X_{l}\right\|_{2}\right)^{2}\right)^{1 / 2} \times\left(\frac{1}{n^{2}} \sum_{k, l=1}^{n}\left\|X_{k}-X_{l}\right\|_{2}^{2}\right)^{1 / 2} \\
& \leq c\left(\frac{1}{n} \sum_{k=1}^{n}\left(\left\|X_{k}^{(p)}-X_{k}\right\|_{2}^{2}\right)^{1 / 2}\left(\frac{1}{n^{2}} \sum_{k, l=1}^{n}\left\|X_{k}-X_{l}\right\|_{2}^{2}\right)^{1 / 2} \stackrel{\text { a.s. }}{\rightarrow} 0 .\right.
\end{aligned}
$$

This proves the lemma. 


\section{Appendix C: Asymptotic behavior under the alternative hypothesis}

In this section we obtain analogs of the previous results under the alternative hypothesis when $X, Y$ are dependent. In this case we need conditions on $X, Y$ which are more restrictive than in the independent case. We investigate the asymptotic behavior of $T_{n, \beta}\left(X^{(p)}, Y^{(p)}\right)$ and $R_{n, \beta}\left(X^{(p)}, Y^{(p)}\right)$ under the alternative. if

In view of (1.4), $T_{\beta}(X, Y), T_{\beta}(X, X)$ and $T_{\beta}(Y, Y)$, hence $R_{\beta}(X, Y)$, are finite

$$
\mathbb{E}\left[\|X\|_{2}^{2 \beta}+\|Y\|_{2}^{2 \beta}\right]<\infty .
$$

Proposition C.1. Assume the following conditions:

1. $X, Y$ are (possibly dependent) stochastically continuous bounded processes on $[0,1]$ defined on the same probability space.

2. If $X, Y$ have finite expectations, then these are assumed to be equal to 0 .

3. $\delta_{n} \rightarrow 0$ as $n \rightarrow \infty$.

4. $\beta \in(0,2)$.

Then the following statements hold.

(1) If either (A1),(A3) or [(B3) and $\left.p \delta_{n}^{\beta+\gamma_{X}^{\prime} \wedge \gamma_{Y}^{\prime}} \rightarrow 0\right]$ hold. Then

$$
T_{n, \beta}\left(X^{(p)}, Y^{(p)}\right)-T_{n, \beta}(X, Y) \stackrel{\mathbb{P}}{\rightarrow} 0,
$$

and

$$
R_{n, \beta}\left(X^{(p)}, Y^{(p)}\right)-R_{n, \beta}(X, Y) \stackrel{\mathbb{P}}{\rightarrow} 0 .
$$

(2) If either (A1), (A3) and

$$
\delta_{n}=o\left(n^{-\frac{1}{(\beta \wedge 1)\left(\gamma X^{\wedge \gamma}\right)}}\right), \quad n \rightarrow \infty,
$$

or (B3) and

$$
\delta_{n}=o\left((p n)^{-\frac{1}{\beta+\gamma_{X}^{\prime} \wedge \gamma_{Y}^{\prime}}}\right), \quad n \rightarrow \infty
$$

hold, then

$$
\sqrt{n}\left(T_{n, \beta}\left(X^{(p)}, Y^{(p)}\right)-T_{n, \beta}(X, Y)\right) \stackrel{\mathbb{P}}{\rightarrow} 0
$$

and

$$
\sqrt{n}\left(R_{n, \beta}\left(X^{(p)}, Y^{(p)}\right)-R_{n, \beta}(X, Y)\right) \stackrel{\mathbb{P}}{\rightarrow} 0 .
$$

Proof. Part (1). First assume that $(X, Y)$ have finite second moment. We follow the lines of proof of Theorem 3.1 from the beginning until inequality (7.3). Again using a symmetry argument, it suffices to consider $I_{11}$. 
Assume $\beta \in(0,1]$. An application of the Cauchy-Schwarz inequality yields

$$
\mathbb{E}\left[I_{11}\right] \leq\left(\mathbb{E}\left[\left|\left\|X_{1}^{(p)}-X_{2}^{(p)}\right\|_{2}^{\beta}-\left\|X_{1}-X_{2}\right\|_{2}^{\beta}\right|^{2}\right]\right)^{1 / 2}\left(\mathbb{E}\left[\left\|Y_{1}^{(p)}-Y_{2}^{(p)}\right\|_{2}^{2 \beta}\right]\right)^{1 / 2} .
$$

By Lyapunov's inequality,

$$
\begin{aligned}
\mathbb{E}\left[\left\|Y_{1}^{(p)}-Y_{2}^{(p)}\right\|_{2}^{2 \beta}\right] & \leq\left(\mathbb{E}\left[\left\|Y_{1}^{(p)}-Y_{2}^{(p)}\right\|_{2}^{2}\right]\right)^{\beta} \\
& \leq c\left(\int_{0}^{1} \operatorname{var}\left(Y^{(p)}(t)\right) d t\right)^{\beta}<\infty .
\end{aligned}
$$

Proceeding as for (7.4) with $\beta / 2$ replaced by $\beta$, we have

$\mathbb{E}\left[\left|\left\|X_{1}^{(p)}-X_{2}^{(p)}\right\|_{2}^{\beta}-\left\|X_{1}-X_{2}\right\|_{2}^{\beta}\right|^{2}\right] \leq \mathbb{E}\left[\left\|\left(X_{1}^{(p)}-X_{2}^{(p)}\right)-\left(X_{1}-X_{2}\right)\right\|_{2}^{2 \beta}\right] \leq c \delta_{n}^{\gamma X \beta}$,

where the condition (A.1) is used.

If $\beta \in(1,2)$, we use the inequality $\left|x^{\beta}-y^{\beta}\right| \leq \beta(x \wedge y)^{\beta-1}|y-x|$ for positive $x, y$ and then the three-function Hölder inequality with conjugates $(2(2 \beta-1) /(\beta-1), 2(2 \beta-1) / \beta, 2)$. This procedure yields

$$
\begin{aligned}
& \mathbb{E}\left[\left|\left\|X_{1}^{(p)}-X_{2}^{(p)}\right\|_{2}^{\beta}-\left\|X_{1}-X_{2}\right\|_{2}^{\beta}\right|\left\|Y_{1}^{(p)}-Y_{2}^{(p)}\right\|_{2}^{\beta}\right] \\
& \leq c \mathbb{E}\left[\left(\left\|X_{1}^{(p)}-X_{2}^{(p)}\right\|_{2}^{\beta-1} \vee\left\|X_{1}-X_{2}\right\|_{2}^{\beta-1}\right)\left\|Y_{1}^{(p)}-Y_{2}^{(p)}\right\|_{2}^{\beta}\right. \\
& \left.\quad \times\left|\left\|\left(X_{1}^{(p)}-X_{2}^{(p)}\right)-\left(X_{1}-X_{2}\right)\right\|_{2}\right|\right] \\
& \leq c\left(\mathbb{E}\left[\left(\left\|X_{1}^{(p)}-X_{2}^{(p)}\right\|_{2}^{2(2 \beta-1)} \vee\left\|X_{1}-X_{2}\right\|_{2}^{2(2 \beta-1)}\right)\right]\right)^{\frac{\beta-1}{2(2 \beta-1)}} \\
& \quad \times\left(\mathbb{E}\left[\left\|Y_{1}^{(p)}-Y_{2}^{(p)}\right\|_{2}^{2(2 \beta-1)}\right]\right)^{\frac{\beta}{2(2 \beta-1)}}\left(\mathbb{E}\left[\left\|\left(X_{1}^{(p)}-X_{1}\right)-\left(X_{2}^{(p)}-X_{2}\right)\right\|_{2}^{2}\right]\right)^{1 / 2} \\
& =c \bar{P}_{1} \cdot \bar{P}_{2} \cdot \bar{P}_{3} .
\end{aligned}
$$

Similarly to the bound for $P_{2}$ in (7.13), we have $\bar{P}_{3} \leq c \delta_{n}^{\gamma_{X} / 2}$ and

$$
\bar{P}_{1}^{\frac{2(2 \beta-1)}{\beta-1}} \leq \mathbb{E}\left[\left\|X_{1}^{(p)}-X_{2}^{(p)}\right\|_{2}^{2(2 \beta-1)}\right]+\mathbb{E}\left[\left\|X_{1}-X_{2}\right\|_{2}^{2(2 \beta-1)}\right]=\bar{P}_{11}+\bar{P}_{12} .
$$

However, $\bar{P}_{11}, \bar{P}_{12}$ are bounded similarly as $P_{11}, P_{12}$ in (7.14). By a symmetry argument, $\bar{P}_{2}$ is bounded. Thus we arrive at $\mathbb{E}\left[\left|I_{1}\right|\right] \leq c \delta_{n}^{\left(\gamma_{X} \wedge \gamma_{Y}\right)(\beta \wedge 1) / 2}$ for $\beta \in$ $(0,2)$, and similar arguments prove

$$
\mathbb{E}\left[\left|I_{2}+I_{3}\right|\right] \leq c \delta_{n}^{\left(\gamma_{X} \wedge \gamma_{Y}\right)(\beta \wedge 1) / 2} .
$$

We omit further details.

Next assume that $X, Y$ have finite $(2 \beta)$ th moment for some $\beta \in(0,1)$. We follow the strategy of the proof in the finite variance case. We only bound $\mathbb{E}\left[\left|I_{1}\right|\right]$ since the quantities $\mathbb{E}\left[\left|I_{i}\right|\right], i=2,3$ can be bounded in a similar manner. Again by the Cauchy-Schwarz inequality,

$$
\mathbb{E}\left[I_{11}\right] \leq\left(\mathbb{E}\left[\left|\left\|X_{1}^{(p)}-X_{2}^{(p)}\right\|_{2}^{\beta}-\left\|X_{1}-X_{2}\right\|_{2}^{\beta}\right|^{2}\right]\right)^{1 / 2}\left(\mathbb{E}\left[\left\|Y_{1}^{(p)}-Y_{2}^{(p)}\right\|_{2}^{2 \beta}\right]\right)^{1 / 2} .
$$


Then direct calculation together with (B3) yields

$$
\begin{aligned}
\mathbb{E}\left[\left\|Y_{1}^{(p)}-Y_{2}^{(p)}\right\|_{2}^{2 \beta}\right] & =\mathbb{E}\left[\left(\int_{0}^{1}\left(Y_{1}^{(p)}(t)-Y_{2}^{(p)}(t)\right)^{2} d t\right)^{\beta}\right] \\
& \leq c \mathbb{E}\left[\max _{0 \leq t \leq 1}|Y(t)|^{2 \beta}\right]<\infty .
\end{aligned}
$$

By concavity and (B3) we have

$$
\begin{aligned}
& \mathbb{E}\left[\left|\left\|X_{1}^{(p)}-X_{2}^{(p)}\right\|_{2}^{\beta}-\left\|X_{1}-X_{2}\right\|_{2}^{\beta}\right|^{2}\right] \\
& \leq \mathbb{E}\left[\left\|\left(X_{1}^{(p)}-X_{1}\right)-\left(X_{2}^{(p)}-X_{2}\right)\right\|_{2}^{2 \beta}\right] \\
& \leq c \mathbb{E}\left[\left(\sum_{i=1}^{p} \int_{\Delta_{i}}\left(\Delta X_{1}\left(t, t_{i}\right]-\Delta X_{2}\left(t, t_{i}\right]\right)^{2} d t\right)^{\beta}\right] \\
& \leq c p \delta_{n}^{\beta+\gamma_{X}^{\prime}} .
\end{aligned}
$$

A symmetry argument yields the corresponding result for $I_{12}$, leading to $\mathbb{E}\left[\left|I_{1}\right|\right] \leq$ $c p^{1 / 2} \delta_{n}^{\left(\beta+\gamma_{X}^{\prime} \wedge \gamma_{Y}^{\prime}\right) / 2}$, and the right-hand side converges to 0 as $n \rightarrow \infty$ by assumption.

Thus we proved, under the assumption of a finite second moment for $X, Y$, that

$$
D_{n}=\mathbb{E}\left[\left|T_{n, \beta}\left(X^{(p)}, Y^{(p)}\right)-T_{n, \beta}(X, Y)\right|\right] \leq c \delta_{n}^{\left(\gamma_{X} \wedge \gamma_{Y}\right)(\beta \wedge 1) / 2},
$$

and, under the assumption of a finite $(2 \beta)$ th moment of $X, Y$ for some $\beta \in(0,1)$, that

$$
D_{n} \leq c p^{1 / 2} \delta_{n}^{\left(\beta+\gamma_{X}^{\prime} \wedge \gamma_{Y}^{\prime}\right) / 2} .
$$

Since the right-hand sides in (C.7) and (C.8) converge to zero by assumption we proved (C.1). The conditions of Lemma 7.2 are satisfied, implying $T_{n, \beta}\left(X^{(p)}, X^{(p)}\right) \stackrel{\mathbb{P}}{\rightarrow} T_{\beta}(X, X), T_{n, \beta}\left(Y^{(p)}, Y^{(p)}\right) \stackrel{\mathbb{P}}{\rightarrow} T_{\beta}(Y, Y)$, and the strong law of large numbers for $V$-statistics yields $T_{n, \beta}(X, X) \stackrel{\mathbb{P}}{\rightarrow} T_{\beta}(X, X), T_{n, \beta}(Y, Y) \stackrel{\mathbb{P}}{\rightarrow}$ $T_{\beta}(Y, Y)$. Then (C.2) follows.

Part (2). Under the growth conditions on $\delta_{n} \rightarrow 0$ we have in both cases, see (C.7) and (C.8), that $\sqrt{n} D_{n} \rightarrow 0$, implying (C.5).

For the convergence in (C.6), we observe that

$$
\begin{aligned}
\sqrt{n} & \left(R_{n, \beta}\left(X^{(p)}, Y^{(p)}\right)-R_{n, \beta}(X, Y)\right) \\
= & \frac{\sqrt{n}\left(T_{n, \beta}\left(X^{(p)}, Y^{(p)}\right)-T_{n, \beta}(X, Y)\right)}{A_{n}} \\
& \quad+\sqrt{n} T_{n, \beta}(X, Y) \frac{T_{n, \beta}(X, X) T_{n, \beta}(Y, Y)-T_{n, \beta}\left(X^{(p)}, X^{(p)}\right) T_{n, \beta}\left(Y^{(p)}, Y^{(p)}\right)}{A_{n} B_{n}},
\end{aligned}
$$


where

$$
\begin{aligned}
& A_{n}=\sqrt{T_{n, \beta}\left(X^{(p)}, X^{(p)}\right) T_{n, \beta}\left(Y^{(p)}, Y^{(p)}\right) T_{n, \beta}(X, X) T_{n, \beta}(Y, Y)}, \\
& B_{n}=\sqrt{T_{n, \beta}\left(X^{(p)}, X^{(p)}\right) T_{n, \beta}\left(Y^{(p)}, Y^{(p)}\right)}+\sqrt{T_{n, \beta}(X, X) T_{n, \beta}(Y, Y)} .
\end{aligned}
$$

The quantities $A_{n}, B_{n}$ converge in probability to positive constants. Therefore it suffices to show that

$$
\begin{aligned}
\sqrt{n}\left(T_{n, \beta}\left(X^{(p)}, X^{(p)}\right)-T_{n, \beta}(X, X)\right) & \stackrel{\mathbb{P}}{\rightarrow} 0, \\
\sqrt{n}\left(T_{n, \beta}\left(Y^{(p)}, Y^{(p)}\right)-T_{n, \beta}(Y, Y)\right) & \stackrel{\mathbb{P}}{\rightarrow} 0,
\end{aligned}
$$

but these relations follow from (C.5) applied to $(X, X)$ and $(Y, Y)$, respectively.

Corollary C.2. Assume the conditions of Proposition C.1. Then $T_{\beta}(X, Y)>0$. Moreover, if $\mathbb{E}\left[\|X\|_{2}^{2 \beta}\|Y\|_{2}^{2 \beta}\right]<\infty$ then the sequence

$$
\sqrt{n}\left(T_{n, \beta}\left(X^{(p)}, Y^{(p)}\right)-T_{\beta}(X, Y)\right)
$$

has a mean-zero Gaussian limit. If also $\mathbb{E}\left[\|X\|_{2}^{4 \beta}+\|Y\|_{2}^{4 \beta}\right]<\infty$ then the sequence

$$
\sqrt{n}\left(R_{n, \beta}\left(X^{(p)}, Y^{(p)}\right)-R_{\beta}(X, Y)\right)
$$

has a mean-zero Gaussian limit.

Proof. We proved in Theorem 4.2 that $T_{\beta}(X, Y)>0$ if and only if $X, Y$ are dependent. In view of Proposition C.1 the statements will follow if we can show that

$$
\sqrt{n}\left(T_{n, \beta}(X, Y)-T_{\beta}(X, Y)\right) \quad \text { and } \quad \sqrt{n}\left(R_{n, \beta}(X, Y)-R_{\beta}(X, Y)\right)
$$

have Gaussian limits. However, the central limit theorem for $T_{n, \beta}(X, Y)$ follows from the fact that it is a non-degenerate $V$-statistic (see the end of this proof) provided it has finite variance; see Arcones and Giné (1992). This condition is ensured by $\mathbb{E}\left[\|X\|_{2}^{2 \beta}\|Y\|_{2}^{2 \beta}\right]<\infty$. It is satisfied due to the assumptions.

As regards the central limit theorem for $R_{n, \beta}(X, Y)$, we can follow an argument similar to the decomposition (C.9). We need to prove joint asymptotic Gaussianity of the vector sequence

$\sqrt{n}\left(T_{n, \beta}(X, Y)-T_{\beta}(X, Y), T_{n, \beta}(X, X)-T_{\beta}(X, X), T_{n, \beta}(Y, Y)-T_{\beta}(Y, Y)\right), \quad n \geq 1$.

This convergence follows if any linear combination of its components has a meanzero Gaussian limit. By virtue of the moment condition $\mathbb{E}\left[\|X\|_{2}^{4 \beta}+\|Y\|_{2}^{4 \beta}\right]<\infty$ each of the components in (C.10) is a non-degenerate $V$-statistic with finite positive variance, hence they have Gaussian mean-zero limits, and if there is joint convergence the limit is non-degenerate. However, any linear combination of 
these components is again a non-degenerate $V$-statistic and therefore the central limit theorem for non-degenerate $V$-statistics with a Gaussian limit applies to them as well.

Finally, we show that the kernel $h$ introduced in Lemma A.1 is non-degenerate, i.e., the conditional expectation $\mathbb{E}\left[h\left(z_{1}, Z_{2}, Z_{3}, Z_{4}\right)\right]$ with deterministic $z_{1}=$ $\left(x_{1}, y_{1}\right)$ and iid random vectors $Z_{i}=\left(X_{i}, Y_{i}\right), i=2,3,4$, is not a constant. By the symmetry of the kernel $h$ in (A.1) we have

$$
\begin{aligned}
\mathbb{E} & {\left[h\left(z_{1}, Z_{2}, Z_{3}, Z_{4}\right)\right] } \\
= & \frac{1}{4} \mathbb{E}\left[f\left(z_{1}, Z_{2}, Z_{3}, Z_{4}\right)+f\left(Z_{2}, z_{1}, Z_{3}, Z_{4}\right)+f\left(Z_{2}, Z_{3}, z_{1}, Z_{4}\right)+f\left(Z_{2}, Z_{3}, Z_{4}, z_{1}\right)\right] \\
= & \frac{1}{4} \mathbb{E}\left[\left(\left\|x_{1}-X_{1}\right\|_{2}^{\beta}\left\|y_{1}-Y_{1}\right\|_{2}^{\beta}+\left\|x_{1}-X_{1}\right\|_{2}^{\beta}\left\|Y_{2}-Y_{3}\right\|_{2}^{\beta}-2\left\|x_{1}-X_{1}\right\|_{2}^{\beta}\left\|y_{1}-Y_{2}\right\|_{2}^{\beta}\right)\right. \\
& \quad+\left(\left\|x_{1}-X_{1}\right\|_{2}^{\beta}\left\|y_{1}-Y_{1}\right\|_{2}^{\beta}+\left\|x_{1}-X_{1}\right\|_{2}^{\beta}\left\|Y_{2}-Y_{3}\right\|_{2}^{\beta}-2\left\|x_{1}-X_{1}\right\|_{2}^{\beta}\left\|Y_{1}-Y_{2}\right\|_{2}^{\beta}\right) \\
& \quad+\left(\left\|X_{1}-X_{2}\right\|_{2}^{\beta}\left\|Y_{1}-Y_{2}\right\|_{2}^{\beta}+\left\|X_{1}-X_{2}\right\|_{2}^{\beta}\left\|y_{1}-Y_{3}\right\|_{2}^{\beta}-2\left\|X_{1}-X_{2}\right\|_{2}^{\beta}\left\|Y_{1}-y_{1}\right\|_{2}^{\beta}\right) \\
& \left.\quad+\left(\left\|X_{1}-X_{2}\right\|_{2}^{\beta}\left\|Y_{1}-Y_{2}\right\|_{2}^{\beta}+\left\|X_{1}-X_{2}\right\|_{2}^{\beta}\left\|y_{1}-Y_{3}\right\|_{2}^{\beta}-2\left\|X_{1}-X_{2}\right\|_{2}^{\beta}\left\|Y_{1}-Y_{3}\right\|_{2}^{\beta}\right)\right] \\
= & \frac{1}{2} \mathbb{E}\left[\left(\left\|x_{1}-X_{1}\right\|_{2}^{\beta}-\left\|X_{1}-X_{2}\right\|_{2}^{\beta}\right)\left(\left\|y_{1}-Y_{1}\right\|_{2}^{\beta}-\mathbb{E}\left[\left\|y_{1}-Y_{1}\right\|_{2}^{\beta}\right]\right)\right] \\
& -\frac{1}{2} \mathbb{E}\left[\left\|X_{1}-x_{1}\right\|_{2}^{\beta}\left(\left\|Y_{1}-Y_{2}\right\|_{2}^{\beta}-\mathbb{E}\left[\left\|Y_{1}-Y_{2}\right\|_{2}^{\beta}\right]\right)\right]+\text { const. }
\end{aligned}
$$

We observe that the kernel

$$
f(x, y)=\|x-y\|_{2}^{\beta}, \quad 0<\beta<2,
$$

is strongly negative definite on $L^{2}[0,1]$ in the sense of Klebanov (2005); see also Lyons (2013), Remark 3.19 and Corollary 3.20. If

$$
\mathbb{E}\left[\left(\left\|x_{1}-X_{1}\right\|_{2}^{\beta}-\left\|X_{2}-X_{1}\right\|_{2}^{\beta}\right)\left(\left\|y_{1}-Y_{1}\right\|_{2}^{\beta}-\mathbb{E}\left[\left\|y_{1}-Y_{1}\right\|_{2}^{\beta}\right]\right)\right]
$$

is independent of $y_{1}$ for any fixed $x_{1}$, then for any $y_{1}$,

$$
\begin{aligned}
& \mathbb{E}\left[\left(\left\|x_{1}-X_{1}\right\|_{2}^{\beta}-\left\|X_{2}-X_{1}\right\|_{2}^{\beta}\right)\left\|y_{1}-Y_{1}\right\|_{2}^{\beta}\right] \\
& \quad=\mathbb{E}\left[\left(\left\|x_{1}-X_{3}\right\|_{2}^{\beta}-\left\|X_{2}-X_{3}\right\|_{2}^{\beta}\right)\left\|y_{1}-Y_{1}\right\|_{2}^{\beta}\right] .
\end{aligned}
$$

We will apply Theorem 4.1 in Klebanov (2005). Note, first of all, that this theorem extends immediately to signed measures. Using this version of the theorem, we have for any Borel set $A \subset L^{2}[0,1]$ and $x_{1} \in L^{2}[0,1]$,

$$
\begin{aligned}
& \mathbb{P}\left(Y_{1} \in A\right) \mathbb{E}\left[\left(\left\|x_{1}-X_{1}\right\|_{2}^{\beta}-\left\|X_{2}-X_{1}\right\|_{2}^{\beta}\right)\right] \\
& \quad=\mathbb{E}\left[\mathbf{1}\left(Y_{1} \in A\right)\left(\left\|x_{1}-X_{1}\right\|_{2}^{\beta}-\left\|X_{2}-X_{1}\right\|_{2}^{\beta}\right)\right] .
\end{aligned}
$$

In the light of Klebanov's Theorem 4.1 we view (C.12), once again, but this time as a function of $x_{1} \in L^{2}[0,1]$. There is a difficulty, though, since there is a 
"free term". However, we can rewrite (C.12) as

$$
\mathbb{E}\left[\mathbf{1}\left(Y_{1} \in A\right)\left\|x_{1}-X_{1}\right\|_{2}^{\beta}\right]=\mathbb{E}\left[\mathbf{1}\left(Y_{1} \in A\right)\left\|x_{1}-X_{3}\right\|_{2}^{\beta}\right]+W,
$$

where

$$
W=\mathbb{E}\left[\mathbf{1}\left(Y_{1} \in A\right)\left\|X_{2}-X_{1}\right\|_{2}^{\beta}\right]-\mathbb{P}\left(Y_{1} \in A\right) \mathbb{E}\left[\left\|X_{2}-X_{1}\right\|_{2}^{\beta}\right] .
$$

Choosing $x_{1}=0$, we see that

$$
W=\mathbb{E}\left[\mathbf{1}\left(Y_{1} \in A\right)\left\|X_{1}\right\|_{2}^{\beta}\right]-\mathbb{P}\left(Y_{1} \in A\right) \mathbb{E}\left[\left\|X_{3}\right\|_{2}^{\beta}\right],
$$

so (C.13) reduces to

$$
\mathbb{E}\left[\mathbf{1}\left(Y_{1} \in A\right)\left(\left\|x_{1}-X_{1}\right\|_{2}^{\beta}-\left\|X_{1}\right\|_{2}^{\beta}\right)\right]=\mathbb{E}\left[\mathbf{1}\left(Y_{1} \in A\right)\left(\left\|x_{1}-X_{3}\right\|_{2}^{\beta}-\left\|X_{3}\right\|_{2}^{\beta}\right)\right] .
$$

If the function $f$ in (C.11) is strongly negative definite on $L^{2}[0,1]$, then so is the function

$$
\tilde{f}(x, y)=\|x-y\|_{2}^{\beta}-\|y\|_{2}^{\beta}, \quad 0<\beta<2 .
$$

Applying Klebanov's theorem to (C.14), we obtain for any Borel set $B \subset L^{2}[0,1]$,

$$
\mathbb{P}\left(Y_{1} \in A, X_{1} \in B\right)=\mathbb{P}\left(Y_{1} \in A, X_{3} \in B\right),
$$

so $X_{1}$ and $Y_{1}$ must be independent, contradicting our assumptions. Therefore the function $\mathbb{E}\left[h\left(z_{1}, Z_{2}, Z_{3}, Z_{4}\right)\right]$ cannot be constant. This concludes the proof.

\section{Acknowledgements}

The paper was finished when Thomas Mikosch visited Ruhruniversität Bochum (RUB) supported by an Alexander von Humboldt Research Award. He would like to thank his colleagues in Bochum for their hospitality. Munyea Matsui visited the University of Copenhagen and RUB, and Laleh Tafakori the University of Copenhagen in 2017/2018 when major parts of this research were developed. They would like to thank their host institutions for hospitality.

Herold Dehling's research was partially supported by the DFG through the Collaborative Research Grant SFB 823. Muneya Matsui's research is partly supported by the JSPS Grant-in-Aid for Young Scientists B (16k16023). Gennady Samorodnitsky's research was partially supported by the ARO grants W911NF12-10385 and W911NF-18 -10318 at Cornell University. Laleh Tafakori would like to thank the Australian Research Council for support through Laureate Fellowship FL130100039.

\section{References}

Arcones, M.A. And Giné, E. (1992) On the bootstrap of $U$ and $V$ statistics. Ann. Statist. 20, 655-674. 
Bickel, P.J. And Freedman, D.A. (1981) Some asymptotic theory for the bootstrap. Ann. Statist. 9, 1196-1217.

Billingsley, P. (1968) Convergence of Probability Measures. Wiley, New York.

Davis, R.A., Matsui, M., Mikosch, T. And Wan, P. (2018) Applications of distance correlation to time series. Bernoulli 24, 3087-3116.

Dehling, H. And Mikosch, T. (1994) Random quadratic forms and the bootstrap for $U$-statistics. J. Multivar. Anal. 51, 392-413.

Feuerverger, A. (1993) A consistent test for bivariate dependence. Int. Stat. Rev. 61, 419-433.

Hoffmann-Jørgensen, J. (1994) Probability with a View Towards Statistics. Chapman \& Hall, New York.

Klebanov, L.B. (2005) $\mathfrak{N}$-Distances and their Applications. Charles University Press, Prague.

Lyons, R. (2013) Distance covariance in metric spaces. Ann. Probab. 41, 32843305.

Lyons, R. (2018) Errata to "Distance covariance in metric spaces". Ann. Probab. 46, 2400-2405.

Marquardt, T. (2006). Fractional Lévy processes with an application to long memory moving average processes. Bernoulli 12, 1099-1126.

Matsui, M., Mikosch, T. and Samorodnitsky G. (2017) Distance covariance for stochastic processes. Probab. Math. Statist. 37, 355-372.

Samorodnitsky, G. (2016) Stochastic Processes and Long Range Dependence. Springer, Berlin.

Samorodnitsky, G. And TAqQU, M.S. (1994) Stable Non-Gaussian Random Processes. Stochastic Models with Infinite Variance. Chapman \& Hall, London.

SzÉKely, G.J., Rizzo, M.L. And Bakirov, N.K. (2007) Measuring and testing dependence by correlation of distances. Ann. Statist. 35, 2769-2794.

SzÉKely, G.J. And Rizzo, M.L. (2009) Brownian distance covariance. Ann. Appl. Stat. 3, 1236-1265.

SzÉKely, G.J. AND Rizzo, M.L. (2013) The distance correlation $t$-test of independence in high dimension. J. Multivar. Anal. 117, 193-213.

SzÉKELY, G.J. AND RIzzo, M.L. (2014) Partial distance correlation with methods for dissimilarities. Ann. Statist. 42, 2382-2412.

SERfling, R.J. (1980) Approximation Theorems of Mathematical Statistics. John Wiley \& Sons, New York. 